\title{
SIMPLE ALGEBRAS AND COHOMOLOGY GROUPS OF ARBITRARY FIELDS
}

\author{
BY \\ S. A. AMITSUR
}

Let $F$ be a finite normal extension of a field $C$ and let $g$ be its Galois group of automorphisms. In the classical theory of simple algebras, it is shown that the Brauer group $\mathfrak{B}(F)$ of all simple algebras over $C$ split by $F$ is isomorphic (canonically) with the second cohomology group $H^{2}\left(F^{*}, \mathcal{S}\right)$ of $\mathcal{G}$ with coefficients in the multiplicative group $F^{*}$ of the nonzero elements of $F$.

If $F$ is a purely inseparable extension of exponent 1 of $C$, then it was shown by $\mathrm{N}$. Jacobson that a Galois theory for such extension is obtained by replacing the Galois group of automorphism of $F$ by the restricted Liealgebra $\mathfrak{L}$ of all derivations of $F$ over $C$. Recently, Hochschild has shown [7] that this Lie algebra can, in some way, replace the Galois group $\mathcal{G}$ in the classical result on the Brauer group $\mathfrak{B}(F)$. More precisely: he has shown that $\mathfrak{B}(F)$ is isomorphic with a certain subgroup of $H_{*}^{2}\left(F^{+}, \mathfrak{l}\right)$ where $H_{*}^{2}\left(F^{+}, \mathfrak{l}\right)$ denotes the second restricted cohomology group of $\mathscr{L}$ with coefficients in the additive group $F^{+}$of the elements of $F$.

Naturally, the question arises now whether these two results are different results, which are connected by the name "cohomology groups" or whether they are two different aspects of one result which includes them. The main purpose of this paper is to answer this question in favor of the second possibility.

A general Galois theory for arbitrary extensions $F$ of $C$ has been obtained by N. Jacobson [10], following some ideas of Kaloujnine, by considering representations of $F$ over $C$. This idea has been followed, in an abstract form, considering $F$-bimodules and the results were extended to other cases by Hochschild [5], Nakayama $[11 ; 12]$ and others. The general theory was obtained by considering certain relatively-cyclic sub-bimodules of the tensor product $F \otimes_{C} F$.

On the other hand, the author has given a method for constructing all simple algebras (in [1]) by dealing with different imbeddings of a field $F$ in a larger field $K$. An extension of this result to arbitrary rings $R$ which is obtained with replacing the field $K$, in particular, by $R=F \otimes{ }_{G} F$, yields a relation between this construction of simple algebras and the general Galois theory. One aspect of this relation will be dealt with elsewhere.

In the present paper we apply the extension of the results of [1] to obtain

Received by the editors March 20, 1957. 
a correspondence between certain algebras over $C$ and relatively-cyclic $\Re\left(V_{F}\right)$-bimodules, where $\Re\left(V_{F}\right)$ is the ring of all linear transformations of an $F$-space $V$. It happens that the central simple algebras are the algebras which correspond to the relatively-cyclic closed bimodules in the sense of JacobsonHochschild (see e.g. [12]). These bimodules form a multiplicative semi-group and it is shown that there exists a homomorphism of this semi-group onto the Brauer group $\mathfrak{B}(F)$.

A certain representation of this semi-group of the relatively-cyclic bimodules leads us to considering a chain-complex $\mathfrak{C}\left(F^{*}\right)$ :

$$
1 \rightarrow F^{*} \rightarrow\left(F \otimes_{C} F\right)^{*} \rightarrow \cdots \rightarrow\left(F \otimes_{C} \cdots \otimes_{C} F\right)^{*} \rightarrow
$$

with a derivation $\Delta$, and where $(*)$ denotes the multiplicative group of the respective ring. For the precise definition of $\Delta$ see $\S 5$. This complex yields the cohomology group $H^{n}\left(\mathfrak{e}\left(F^{*}\right)\right)=$ Kernel $\Delta /$ Image $\Delta$, and our main result is that: $H^{2}\left(\mathfrak{e}\left(F^{*}\right)\right) \cong \mathfrak{B}(F)$.

Furthermore, we prove that this result includes the classical result of Brauer and a parallel result to the recent result of Hochschild quoted above. Actually we prove that if $F$ is a normal extension of $C$ with the Galois group $\mathcal{G}$ then $H^{n}\left(\mathfrak{e}\left(F^{*}\right)\right) \cong H^{n}\left(F^{*}, \mathcal{G}\right)$, and if $F$ is a pure inseparable extension of $C$ of exponent 1 with the Lie algebra of derivation $\mathscr{L}$ then $H^{n}\left(\mathbb{C}\left(F^{*}\right)\right)$ is isomorphic with a certain subgroup of $H_{*}^{n}\left(F^{+}, \mathfrak{L}\right)$, which is probably (but has not been shown yet) for $n=2$ identical with the group of Hochschild.

The paper contains two parts: Part I includes $\$ \$ 1-4$ deals with the extension of Part I of [1] to rings which satisfy certain conditions. In the second part, which contains $\S \S 5-8$, we define the general cohomology groups of arbitrary fields $F$ and prove the results stated above. The last section contains an extension of a result of Hochschild [8] regarding the behavior of the Brauer group over fields of characteristic $p$ under pure inseparable extensions.

1. Semi-linear transformations. Let $S$ be a subring of a commutative ring $R$. Let $R$ and $S$ contain the same identity element. We shall consider the ring $R$ both as a right and as a left $S$-module and we assume that $R$ is an $S$ free module.

Let $V$ be a (left) $R$-module and let $W$ be an $S$-admissible subgroup of $V$ (i.e., $s W \subset W$ for all $s \in S$ ). We shall say that $W$ is a regular $S$-submodule of $V$ is the homomorphic mapping $\mu: R \otimes_{S} W \rightarrow V$ defined by $\mu(r \otimes v)=r v$, $r \in R$ and $v \in W$, is an isomorphism.

We note that if $V$ contains a regular $S$-submodule $W$ which is $S$-free, then since $\mu$ is an isomorphism it follows that $S$-independent elements of $W$ are also $R$-independent in $V$; furthermore, $V$ itself is a free $R$-module.

If $W$ is a left module over a ring $K$, we shall denote by $\Re_{K}(W)$ the ring of all $K$-endomorphisms of $W$. The notation $\Re(W)$ will be used instead of $\Re_{K}(W)$ when no confusion about the ring $K$ is involved. 
Let $W$ be a free regular $S$-submodule of $V$, then clearly: $\Re_{R}\left(R \otimes_{S} W\right)$ $=R \otimes_{S} \Re_{S}(W)$ and, thus, $\mu$ induces an isomorphism between the ring $R \otimes_{S} \Re_{S}(W)$ and $\Re_{R}(V)$ and we shall often identify the two rings.

In this paper we shall be interested in the following situation: Let $S=F$ be a subfield of a commutative ring $R$ and let $C$ be a fixed subfield of $F$. In what follows we assume that: $R$ contains a nilpotent ideal $N$ (which may be zero) such that $R / N$ is a direct sum of a finite number of fields.

In this case every $F$-module is free, and thus the requirements of the preceding remarks hold. Let $V_{F}$ be an $F$-space of dimension $n$ and let $V=R \otimes_{F} V_{F}$. Clearly, $V_{F}$ is a regular $F$-subspace of $V$ and, therefore, it follows by the preceding remarks that $\Re_{R}(V)=R \otimes_{F} \Re_{F}\left(V_{F}\right)$. We shall use the notations $\Re(V), \mathfrak{R}\left(V_{F}\right)$ instead of $\Re_{R}(V)$ and $\Re_{F}\left(V_{F}\right)$ respectively. Thus, these rings denote the ring of all linear transformations of the spaces $V$ and $V_{F}$ respectively.

Let $\phi$ be an isomorphism of $F$ into $R$ which leave the elements of the subfield $C$ invariant.

Definition 1. A ( $\phi$-) semi-linear transformation $T$ of $V_{F}$ into $V$ is a homomorphism $T$ of $V_{F}$ into $V$ which satisfies: $\left.{ }^{1}\right)$

$$
T(h v)=h^{\phi} T v \quad h \in F \text { and } v \in V_{F} .
$$

Since $F^{\phi}=\left\{h^{\phi} ; h \in F\right\} \subseteq R$ is a field, the set $T V_{F}=\left\{T v, v \in V_{F}\right\}$ is an $F^{\phi}$-subspace of $V$ and clearly $\left(T V_{F}: F^{\phi}\right) \leqq n$.

Definition 2. A $\phi$-semi-linear transformation $T$ of $V_{F}$ into $V$ is said to be regular if the space $T V_{F}$ is a regular subspace of $V$. i.e., if the natural mapping $\left(^{*}\right) \mu: R \otimes_{F^{\phi}} T V_{F} \rightarrow V$, defined by $\mu(r \otimes T v)=r T v$, is an isomorphism.

If $T$ is regular, then clearly $T$ is an isomorphism of $V_{F}$ onto $T V_{F}$; furthermore, if the set $\left(v_{i}\right)$ is an $F$-base of $V_{F}$ then the set $\left(T v_{i}\right)$ will be an $R$-base of $V$ as well as an $F^{\phi}$-base of $T V_{F}$. Conversely, the most general regular semilinear transformation $T$ of $V_{F}$ into $V$ is obtained by choosing an $F$-base $\left(v_{i}\right)$ of $V_{F}$ and an $R$-base $\left(w_{i}\right)$ of $V$ and defining: $T\left(\sum h_{i} v_{i}\right)=\sum h_{i}^{\phi} w_{i}$, for all $h_{i} \in F$.

In [1, Part I] we have considered only semi-linear transformations in vector spaces over fields, i.e., the case $R=K$ is a field. We now extend the theory of that paper to an arbitrary commutative ring satisfying the requirements mentioned in the beginning of this section. Almost all of the results of $[1$, Part I $]$ can be carried over to the present case without any additional efforts. We shall quote the results of [1] which will be needed in the context and we include new proofs only in those cases where the fact that $R$ is not a field affects the proof given in [1].

LemMa 1.1. If $T$ is a regular semi-linear transformation of $V_{F}$ into $V$ and $P, Q$ are regular linear transformations of $V_{F}$ and $V$ respectiveiy, then $Q T P$ is also a regular semi-linear transformation of $V_{F}$ into $V$ [1, Lemma 1.1].

(1) Operators of a ring or of a space will be written multiplicatively on the left of the element on which they operate. 
Since $T$ is an isomorphism, it follows readily that $T \Re\left(V_{F}\right) T^{-1}=\Re_{F^{\phi}}\left(T V_{F}\right)$ and, therefore, for any $P \in \Re\left(V_{F}\right)$ the element: $\mu\left(1 \otimes T P T^{-1}\right)=P^{T}$ belongs to $\Re(V)$. Thus, we have:

$$
P^{T} T v=\mu\left(1 \otimes T P T^{-1}\right)(1 \otimes T v)=\mu(1 \otimes T P v)=T P v, \quad v \in V_{F},
$$

which follows by the definition of $\mu$ and by the relation between $\Re(V)$ and $\Re\left(V_{F}\right)$. This proves that $P^{T} T=T P$ and clearly this relation determines $P^{T}$ uniquely.

Lemma 1.2. If $T$ is a regular semi-linear transformation of $V_{F}$ into $V$, then for any linear transformation $P \in \Re\left(V_{F}\right)$ there exists a unique linear transformation $P^{T} \in \Re(V)$ such that $T P=P^{T} T$, and the correspondence $P \rightarrow P^{T}$ is a $\phi$-extension isomorphism of $\Re\left(V_{F}\right)$ into $R(V)$, i.e., $h^{T}=h^{\phi}$ [1, Lemma 1.2].

Lemma 1.3. Let $T$ be a regular s.l.t. of $V_{F}$ into $V$, then $\Re(V) T$ is the set of all semi-linear transformations of $V_{F}$ into $V$. Furthermore, a semi-linear transformation $S=Q T P, Q \in \Re(V), P \in \Re\left(V_{F}\right)$ is regular, if and only if $P$ and $Q$ are regular linear transformations, and then $A^{S}=Q\left(P A P^{-1}\right)^{T} Q^{-1}$.

This lemma is included in [1, Lemma 1.3]. We recall that if $T_{1}$ and $T$ are semi-linear transformations of $V_{F}$ into $V$ and $T$ is regular, then $T_{1}=Q T$ where $Q$ is defined by the relation $Q\left(T v_{i}\right)=T_{1} v_{i}$, for a given base $\left\{v_{i}\right\}$ of $V_{F}$.

Let $V, V_{F}$ and $W, W_{F}$ be two spaces over $R$ and $F$ of the type considered above and let $T, S$ be regular semi-linear transformations of $V_{F}$ into $V$ and of $W_{F}$ into $W$ respectively. Then the definition $(T \otimes S)(v \otimes w)=T v \otimes S w$ yields a regular semi-linear transformation of $V_{F} \otimes_{F} W_{F}$ into $V \otimes_{R} W$.

DeFinition 3. The set of all linear transformations $P$ of $V_{F}$ which commute with a regular semi-linear transformation $T$ is called the invariant ring of $T$, and will be denoted by $O(T)$.

That is: $O(T)=\left\{P ; P \in \Re\left(V_{F}\right), P^{T}=P\right\}$.

From Lemma 1.3 it follows immediately that:

Corollary 1.1. If $S=P^{-1} T P, P \in R\left(V_{F}\right)$ then $O(S)=P^{-1} O(T) P$.

The main result of $[1$, Part I] which is fundamental also for the present paper is the following:

Theorem 1.1. Let $\mathfrak{A}$ be a central simple algebra of order $n^{2}$ over $C$ which is split by $F$, then there exists a regular semi-linear transformation $T$ of $V_{F}$ into $V$ such that $\mathfrak{A}$ is isomorphic with a subring of $O(T)$. Furthermore, $O(T) \cong \mathfrak{A} \otimes_{C} D$, where $D=\left\{h ; h \in F, h^{\phi}=h\right\}$.

The proof of this theorem in [1] depends on the fact that $R$ is a field and $V$ is a vector space over a field. Before proceding with the proof for commutative rings $R$ satisfying the condition stated above, we begin with some lemmas. 
Lemma 1.4. Let $R$ be a primary ring $\left({ }^{2}\right)$ and $\mathfrak{A}$ a central simple algebra of order $n^{2}$ over $C$. If $\mathfrak{A}$ has a representation in the ring $\mathfrak{R}(V)$ of linear transformations of $V$, then $\mathfrak{N}(V) \cong \mathfrak{A} \otimes_{C} R$.

Proof. Let $\mathfrak{A}_{1}$ be the subring of $\mathfrak{R}(V)$ which is isomorphic with $\mathfrak{A}$. Since $\mathfrak{A}_{1}$ is a simple ring and finite over $C$ it follows that $\Re(V)=\mathfrak{A}_{1} \otimes_{C} R_{1}$, where $R_{1}$ is the centralizer of $\mathfrak{A}_{1}$ in $\Re(V)$. Let $\left\{a_{i}\right\}$ be a base of $\mathfrak{A}_{1}$ and $\left\{e_{i}\right\}$ a base of $\Re(V)$ over $R$; for both bases we have $i=1,2, \cdots, n^{2}$. Hence,

$$
a_{i}=\sum r_{i k} e_{k}, \quad r_{i k} \in R .
$$

Let $N_{v}$ be the radical $\left(^{3}\right)$ of $\Re(V)$ and let $\overline{\mathfrak{A}}_{1}$ be the representation of $\mathfrak{A}$ in $\Re(V) / N_{v}=\bar{\Re}(V)$. Clearly, $\bar{\Re}(V)$ is isomorphic with a complete matrix ring over the field $\bar{R}=R / N$, where $N$ is the radical of $R$. Since $\overline{\mathfrak{A}}_{1}=\left(\mathfrak{R}_{1}, N_{v}\right) / N_{v}$ and $\mathfrak{A}_{1} \Phi N_{v}$, it follows that $\overline{\mathfrak{A}}_{1} \neq 0$. Furthermore, $\overline{\mathfrak{A}}_{1} \otimes \bar{R} \subseteq \bar{\Re}(V)$ and $\bar{R}$ $\subseteq\left(R_{1}, N_{v}\right) / N_{v}$. Now, since $\left(\overline{\mathfrak{A}}_{1}: C\right)=(\bar{\Re}(V): \bar{R})=n^{2}$ and $\bar{R}$ is a field, we must have $\overline{\mathfrak{A}}_{1} \otimes \bar{R}=\bar{\Re}(V)$. Hence, (1.1) yields that $\bar{a}_{i}=\sum \bar{r}_{i k} \bar{e}_{k}$ and $\left(\bar{r}_{i k}\right)$ is a regular matrix of order $n^{2}$ over $\bar{R}$. Let $\left(\bar{s}_{i k}\right)=\left(\bar{r}_{i k}\right)^{-1}$ and let $\left(s_{i k}\right)$ be any matrix such that the elements $s_{i k}$ represent the class $\bar{s}_{i k} \bmod N$. Since $\left(\bar{s}_{i k}\right)\left(\bar{r}_{i k}\right)=\overline{1}$, it follows that $\left(s_{i k}\right)\left(r_{i k}\right)=1+n$ and $n$ is a matrix with coefficients in $N$ and, therefore, it is nilpotent, say $n^{r}=0$. Then $1+n$ is regular and its inverse is $1-n+\cdots+(-1)^{r} n^{r}$. Consequently, the matrix $\left(r_{i k}\right)$ is regular in $R$. From this we conclude by (1.1) that the elements of the base $\left\{e_{i}\right\}$ are linear combinations of the elements $\left\{a_{i}\right\}$ with coefficients in $R$. Hence $\mathfrak{A}_{1} \otimes R \supseteq \Re(V)$. This readily yields that $\mathfrak{A}_{1} \otimes_{C} R=R(V)$, which proves the lemma.

We shall need also a classical result of Artin [3]( $\left.{ }^{4}\right)$.

Lemma 1.5. If $R$ is a primary ring then the automorphisms of $\Re(V)$ which leave the elements of $R$ invariant are inner.

For completeness sake we outline a proof of this lemma: Let $\left\{c_{i k}\right\}$ be an orthogonal base of $R(V)$, i.e.; $c_{i k} c_{j k}=\delta_{k j} c_{i k}$; and let $\bar{c}_{i k}$ be the elements corresponding to $c_{i k}$ under a given automorphism. Since $R(V)$ is also primary one verifies that $c_{11} R(V) \bar{c}_{11} \cdot \bar{c}_{11} R(V) c_{11}=c_{11} R(V) c_{11}$. Hence, $c_{11} R(V) \bar{c}_{11}$ contains a non-nilpotent element $a$. Since $a \bar{c}_{11} R(V) c_{11}$ is again a potent two-sided ideal in $c_{11} R(V) c_{11}$ which is a primary ring, it follows that $a \bar{c}_{11} R(V) c_{11}=c_{11} R(V) c_{11}$. Thus, there exists $b \in \bar{c}_{11} R(V) c_{11}$ such that $a b=c_{11}$. One then verifies that the required automorphism is the inner automorphism determined by the element $q=\sum_{\lambda} \bar{c}_{\lambda 1} b c_{1 \lambda},\left(q^{-1}=\sum_{\lambda} c_{\lambda 1} a \bar{c}_{1 \lambda}\right)$.

(2) A ring $R$ with a unit is said to be a primary ring, if $R / N$ is a field and $N$ is the maximal nilpotent ideal in $R$.

(3) By the radical we mean the maximal nilpotent ideal.

(4) See also, Theorem 3 Chapter III, p. 59 in the book: N. Jacobson, Structure of rings, Amer. Math. Soc. Colloquium Publications vol. 37, 1956. 
Lemma 1.6. If $\mathfrak{A}$ is a central simple algebra of order $n^{2}$ over $C$, then any two representations of $\mathfrak{A}$ in $\mathfrak{R}(V)$ are similar.

This result is well known for simple or semi-simple ring $\Re$. To prove this lemma for arbitrary rings $R$ of the type considered in the present section, we begin with the case where $R$ is a primary ring.

Let $\mathfrak{A}_{1}, \mathfrak{A}_{2}$ be the two representations of $\mathfrak{A}$. Thus, $\mathfrak{A}_{1} \cong \mathfrak{H}_{2} \cong \mathfrak{A}$. It follows by the proof of Lemma 1.4 that $\mathfrak{A}_{1} \otimes R=\mathfrak{A}_{2} \otimes R=\mathfrak{R}(V)$. Hence, the isomorphism between $\mathfrak{A}_{1}$ and $\mathfrak{H}_{2}$ can be extended to an automorphism of $\mathfrak{R}(V)$ leaving the elements of $R$ invariant. It follows, therefore, by the preceding lemma that this automorphism is an inner automorphism. Consequently, the two representations are similar.

Consider now the general case where $R$ is a commutative ring with an identity and a radical $N$ such that $R / N$ is a direct sum of a finite number of fields. Let $1=e_{1}+\cdots+e_{m}$ be the decomposition of the unit into a sum of primitive orthogonal idempotents. Then $e_{i} R e_{i}$ are primary rings with the radical $e_{i} N e_{i}$. By considering $R$ as a subring of $\Re(V)$, we obtain that the $e_{i}$ are also central idempotents of $\Re(V)$ and that the $e_{i} \Re(V) e_{i}$ are isomorphic with complete matrix rings of order $n^{2}$ over $e_{i} R e_{i}$. Let $\mathfrak{A}_{1}, \mathfrak{A}_{2}$ be two representations of $\mathfrak{A}$ in $\mathfrak{N}(V)$ then, clearly, $e_{i} \mathfrak{A}_{1} e_{i}$ and $e_{i} \mathfrak{A}_{2} e_{i}$ are two representations of $\mathfrak{A}$ in $e_{i} \Re(V) e_{i}$. By the first part of the proof, these two representations are similar in $e_{i} \Re(V) e_{i}$. That is: $e_{i} \mathfrak{A}_{1} e_{i}=p_{i} e_{i} \mathfrak{A}_{2} e_{i} p_{i}^{-1}$ for $p_{i} \in e_{i} \Re(V) e_{i}$ and $p_{i} p_{i}^{-1}=e_{i}$ which is the unit of $e_{i} \Re(V) e_{i}$. Put $p=p_{1}+\cdots+p_{m}$ then $p_{i} p_{k}=0$ for $i \neq k$ and $p^{-1}=p_{1}^{-1}+\cdots+p_{m}^{-1}$, and it follows readily that $p \mathfrak{A}_{2} p^{-1}=\mathfrak{H}_{1}$. q.e.d.

The preceding method of reducing the general case to the primary one yields also the following extension of Lemma 1.4.

Lemma 1.4.* Let $\mathfrak{A}$ satisfy the condition of Lemma 1.4 and let $R$ be an arbitrary ring of the type considered above. If $\mathfrak{A}$ has a representation $\mathfrak{A}_{1}$ in $\Re(V)$ then $\mathfrak{A}_{1} \otimes{ }_{G} R=\mathfrak{R}(V)$.

This immediately yields:

Corollary 1.2. If $A_{1}, \cdots, A_{n}$ is a C-base of $\mathfrak{A}_{1}$, then it is also an $R$-base of $\Re(V)$.

We can now turn to the proof of Theorem 1.1. The proof runs similarly to the proof of [1, Theorem 5.3] with Lemma 1.6 replacing [1, Theorem 5.2]. More precisely: since $F$ splits $\mathfrak{A}$, the latter has a representation $\mathfrak{A}_{1}$ in $R\left(V_{F}\right)$. Now, let $S$ be any regular semi-linear transformation of $V_{F}$ into $V$, then the mapping $A \rightarrow A^{S}$ for $A \in \mathfrak{A}_{1}$ determines another representation $\mathfrak{A}_{1}^{S}$ of $\mathfrak{A}$ in $\Re(V)$. It follows, by Lemma 1.6 , that $A^{S}=P^{-1} A P$ for every $A \in \mathfrak{A}_{1}$ and some regular $P \in \Re(V)$. Put $T=P S$, then from Lemma 1.3 we have: $A^{T}=P A^{S} P^{-1}$ $=A$, for all $A \in \mathfrak{A}_{1}$. This means that $\mathfrak{A}_{1} \subseteq O(T)$.

The invariant field $D$ of the theorem clearly belongs to $O(T)$ and by Corollary 1.2 it follows that $\mathfrak{A}_{1}$ possesses an $R$-base of $\Re(V)$, hence $\mathfrak{A}_{1} \otimes D$ 
$\subseteq O(T)$. The rest follows now by [1, Lemma 3.3] and [1, Corollary 3.1] which holds also in our case. We remark that the proof of [1, Lemma 3.3] holds without any change for the general ring $R$ by noting that if a set of linear equations with coefficients in $F$ has a unique solution in the ring $R(\supseteq F)$ then this solution is necessarily in $F$.

Following the notations of [1], we shall say that a semi-linear transformation $T$ represents the central simple algebra $\mathfrak{A}$ if $\mathfrak{A} \otimes D=O(T)$.

Further results which will be needed later are:

TheOREm 1.2. If $T$ represents $\mathfrak{A}$ and $\mathbf{S}$ represents $\mathfrak{B}$ then $\mathfrak{A} \otimes D \cong \mathfrak{B} \otimes D$ if and only if $T=t P S P^{-1}$ for some regular element $t \in R$ and a regular linear transformation $P \in \Re\left(V_{F}\right)$.

For if $\mathfrak{A} \otimes D \cong \mathfrak{B} \otimes D$, then $O(\boldsymbol{T})$ and $O(\boldsymbol{S})$ are two representations of this algebra in $\Re\left(V_{F}\right)$. Hence, $P^{-1} O(S) P=O(T)$ for some regular linear transformation $P \in \Re\left(V_{F}\right)$. Let $S_{1}=P^{-1} S P$, then Corollary 1.1 implies that $O\left(S_{1}\right)$ $=P^{-1} O(\boldsymbol{S}) P=O(\boldsymbol{T})$.

A second application of Lemma 1.3 yields that $T=P_{1} S_{1}$, where $P_{1}$ is a regular linear transformation of $V$, and $Q^{T}=P_{1} Q^{S_{1}} P_{1}^{-1}$. In particular for $Q \in O(T)=O\left(S_{1}\right)$, we have $Q=P_{1} Q P_{1}^{-1}$ which means that $P_{1}$ belongs to the centralizer of $O(T)$ in $\Re(V)$. It follows, by Lemma $1.4^{*}$, that $R$ is the centralizer of $O(T)$. Hence, $P_{1}=t \in R$ and, therefore, $T=t S_{1}=t P^{-1} S P$.

Let $V_{0}$ be a fixed vector space over the field $C$ such that $V=R \otimes_{C} V_{0}$ and $V_{F}=F \otimes_{C} V_{0}$. Let $E$ be the injection of $V_{0}$ into $V_{F}$, then $E$ can be extended uniquely to a $\phi$-semi-linear transformation of $V_{F}$ into $V$ by setting: $E\left(h \otimes v_{0}\right)$ $=h^{\phi} E v_{0}=h^{\phi} v_{0}$, for every $h \in F$ and $v_{0} \in V_{0}$. Then, clearly, $O(E)$ is isomorphic with a complete matrix ring over $C$. Thus, the last theorem yields:

COROLlary 1.3. $T$ represents a central simple algebra which is split by $D$ if and only if $T=t P^{-1} E P, t \in R, P \in \Re\left(V_{F}\right)$ are regular.

The theory of invariant submodules and homomorphic images of regular semi-linear transformations can be carried over to our case where $R$ is a general commutative ring, by a slight change in the definition of invariant submodules; and this we intend to carry out now:

Let $V_{F}$ be a regular $F$-subspace of $V$. An $R$-subspace $W$ of $V$ is said to be a regular subspace of $V$, if $W \cap V_{F}$ is a regular $F$-subspace of $W$. This is equivalent to saying that $W \cap V_{F}$ contains an $F$-base $v_{1}, \cdots, v_{r}$ such that $W=R v_{1} \oplus \cdots \oplus R v_{r}$. In other words: $W \cong R \otimes_{F}\left(W \cap V_{F}\right)$.

Let $T$ be a regular semi-linear transformation of $V_{F}$ into $V$. An $R$-subspace $W \subseteq V$ is said to be a $(\boldsymbol{T}-)$ invariant subspace if $W$ is a regular subspace of $V$ in the preceding sense and $\boldsymbol{T}\left(W \cap V_{F}\right) \subseteq W$.

With this definition one can carry the proofs of the results of $[1$, Lemmas $1.4,1.5$ and 1.6] to the general case after noting the following fact:

Lemma 1.7. If $W$ and $U$ are regular subspaces of $V$ then so are $W \cup U$ and 
$W \cap U$. If $\pi$ is a homomorphism of $V$ onto $W$ such that the kernel of $\pi$ is a regular subspace $V_{0}$ of $V$ then $\pi$ induces a lattice isomorphism between the regular subspaces of $V$ containing $V_{0}$ and the regular subspaces of $W$.

The proof of this lemma is very similar to the proof [1, Lemma 1.5] but one avoids that part of the proof in [1] which deals with the dimensions of

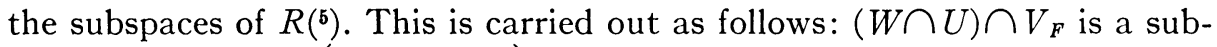
space of $V_{F}$ so let $\left\{v_{1}, \cdots, v_{r}\right\}$ be an $F$-basis of this subspace. Complete this base to a base $\left\{v_{1}, \cdots, v_{r}, v_{1}^{\prime}, \cdots, v_{u}^{\prime}\right\}$ of $U \cap V_{F}$ and to a base $\left\{v_{1}, \cdots, v_{r}, v_{1}^{\prime \prime}, \cdots, v_{v}^{\prime \prime}\right\}$ of $W \cap V_{F}$. Since $U$ and $W$ are regular $\left\{v_{i}, v_{j}^{\prime}\right\}$ and $\left\{v_{i}, v_{j}^{\prime \prime}\right\}$ are an $R$-base of $U$ and $W$, respectively. The set $\left\{v, v^{\prime}, v^{\prime \prime}\right\}$ is $F$-independent; for if $\sum h v+\sum h^{\prime} v^{\prime}+\sum h^{\prime \prime} v^{\prime \prime}=0, h, h^{\prime}, h^{\prime \prime} \in F$, then w $=-\sum h^{\prime \prime} v^{\prime \prime}=\sum h v+\sum h^{\prime} v^{\prime} \in\left(W \cap V_{F}\right) \cap\left(U \cap V_{F}\right)=(W \cap U) \cap V_{F}$, but $\left\{v_{i}\right\}$ is a base of the latter; hence $h^{\prime \prime}=0$ and, consequently, $h^{\prime}=h=0$. Now, $V_{F}$ is a regular $F$-subspace of $V$, hence $\left\{v, v^{\prime}, v^{\prime \prime}\right\}$ are also $R$-independent. Let $w \in W \cap U$, then $w=\sum r_{i} v_{i}+\sum r_{i}^{\prime} v_{i}^{\prime}$ and $w=\sum s_{i} v_{i}+\sum r_{i}^{\prime} v_{i}^{\prime \prime}, s_{i}, r_{i}, r_{i}^{\prime}, r_{i}^{\prime \prime}$ $\in R$, and, therefore, we have $r_{i}=s_{i}$ and $r_{i}^{\prime}=r_{i}^{\prime \prime}=0$. From this it follows readily that $\left[(W \cap U) \cap V_{F}\right] \otimes R=W \cap U$ which means that $W \cap U$ is a regular subspace of $V$. Furthermore, the set $\left\{v, v^{\prime}, v^{\prime \prime}\right\}$ is an $R$-base of $W \cup U$ and, clearly, it is also an $F$-base of $\left(W \cap V_{F}\right) \cup\left(U \cap V_{F}\right)$ which is contained in $(W \cup U) \cap V_{F}$. Complete it to an $F$-base of $V_{F}$ which is necessarily also a base of $V$. If $w \in(W \cup U) \cap V_{F}$, then it follows that $w=\sum r v+\sum r^{\prime} v^{\prime}+\sum r^{\prime \prime} v^{\prime \prime}$, $r, r^{\prime}, r^{\prime \prime} \in R$ and it can also be expressed as a linear combination of $\left\{v, v^{\prime}, v^{\prime \prime}\right\}$ and other $R$-independent elements with coefficients in $F$. Hence, necessarily $r, r^{\prime}, r^{\prime \prime} \in F$. This means that $W \cup U$ is also a regular subspace of $V$. The rest of the lemma follows similarly.

With these results we can carry over all the rest of part I of [1]. In particular for later references we quote the following theorems which are equivalent to $[1$, Theorem 5.8] and [1, Theorem 5.6]:

TheоRem 1.3. Let $T$ represent a central simple algebra and let $\mathrm{M}: V \rightarrow W$ be a homomorphism of $V$ onto $W$ such that $\mathrm{M} T=\mathrm{SM}$ for some regular semi-linear transformation $\mathbf{S}$ of $W$, then $O(T)$ and $O(S)$ are similar algebras in the sense of $\operatorname{Brauer}\left({ }^{6}\right)$.

THEOREM 1.4. If $T$ represents a central simple algebra $\mathfrak{A}$ in $V$ and $\mathbf{S}$ represents a central simple algebra $\mathfrak{B}$ in $W$, then $T \otimes S$ is a regular semi-linear transformation in $V \otimes_{R} W$ and it represents $\mathfrak{H} \otimes \mathfrak{B}$. Actually, we have $O(T) \otimes O(S)$ $=O(T \otimes S)$.

We recall that $T \otimes S$ was defined by the relation $(T \otimes S)(v \otimes w)=T v \otimes S w$ for $v \in V_{F}, w \in W_{F}$.

(5) $R=F$ in [1].

(6) Two central simple algebras $\mathfrak{A}$ and $\mathfrak{B}$ over $C$ are said to be similar if $\mathfrak{A} \cong \mathfrak{C}_{n}, \mathfrak{B} \cong \mathfrak{C}_{m}$ for some central simple algebra $\mathfrak{C}$. 
2. Double modules and semi-linear transformations. In [1] we gave a characterisation for a semi-linear transformation to represent a central simple algebra. We follow here a different and more applicable approach. This approach utilises the notions of double modules and relatively cyclic double modules as introduced by Hochschild [5] and later developed for a more general case by Nakayama [12]. In the present paper we shall follow the method of Nakayama, and we recall the following notations and definitions of [12] with some slight changes.

Let $S$ be a ring and let $\mathfrak{M}$ be an $S$-bimodule; i.e., $\mathfrak{M}$ is both a right and left $S$-module and $\left(s_{1} m\right) s_{2}=s_{1}\left(m s_{2}\right)$ for $m \in \mathfrak{M}, s_{i} \in S$. By a homomorphism of an $S$-bimodule $\mathfrak{M}$ we shall mean a homomorphism of $\mathfrak{M}$ onto an $S$-bimodule which preserves the operators of $S$ both on the right and on the left.

By a relatively cyclic $S$-bimodule we mean a pair $(\mathfrak{M}, A)$ consisting of an $S$-bimodule $\mathfrak{M}$ and an element $A \in \mathfrak{M}$. A relatively cyclic bimodule $(\mathfrak{M}, A)$ is said to cover $(\mathfrak{N}, B)$, which is denoted by $(\mathfrak{M}, A) \geqq(\mathfrak{N}, B)$, if there exists a homomorphism $\phi: \mathfrak{M} \rightarrow \mathfrak{N}$ of $\mathfrak{M}$ onto $\mathfrak{N}$ such that $\phi(A)=B$.

The sum and the product of two relatively cyclic $S$-bimodules $(\mathfrak{M}, A)$ and $(\mathfrak{N}, B)$ are defined as follows:

$$
\begin{aligned}
(\mathfrak{M}, A)+(\mathfrak{N}, B) & =(\mathfrak{M} \oplus \mathfrak{N}, A \oplus B), \\
(\mathfrak{M}, A)(\mathfrak{N}, B) & =\left(\mathfrak{M} \otimes_{S} \mathfrak{N}, A \otimes B\right)
\end{aligned}
$$

where the product $\mathfrak{M} \otimes \mathfrak{N}$ is taken relatively to $S$; i.e., $m \otimes P n=m P \otimes n$ for $m \in \mathfrak{M}, n \in \mathfrak{N}$ and $P \in S$.

A relatively cyclic bimodule $(\mathfrak{M}, A)$ is said to be regular if:

(2.1) $A s=0, s \in S$ implies $s=0$

(2.2) The module $S A S$ has a left $S$-base consisting of elements of the form $s_{i} A, s_{i} \in S$.

That is, $S A S=\sum s_{i} A S$, and the sum is direct.

The relatively cyclic bimodule $(\mathfrak{M}, A)$ is said to be closed if $(\mathfrak{M}, A)$ $\geqq(\mathfrak{M}, A)(\mathfrak{M}, A)$.

Let $\&$ denote the set of all $\phi$-semi-linear transformations of $V_{F}$ into $V$. We shall consider the set $\mathfrak{R}$ as an $\Re\left(V_{F}\right)$-bimodule with the multiplication by elements of $\Re\left(V_{F}\right)$ being defined in the usual way of product of transformations. That is: $(T P) v=T(P v)$ and $(P T) v=P(T v)$ for all $v \in V_{F}$, and $P \in \Re\left(V_{F}\right)$. Clearly, $P T, T P \in \mathbb{R}$.

We note also that the fact that $P \in \Re\left(V_{F}\right)$ was used only in the definition of $T P$, whereas $P T$ may be defined for all $P \in \Re(V)$. Hence $L$ is also a left $\Re(V)$-module.

For the rest of this paper we shall denote by $R$ the ring $R=F \otimes_{G} F$. This ring contains two isomorphic images of $F$; the first, is the set of all elements $\{h \otimes 1 ; h \in F\}$ and the second is the set $\{1 \otimes h ; h \in F\}$. We identify, henceforth, the elements $h \otimes 1$ with $F$, and thus $F$ is assumed to be a subfield of $R$. We shall put $h^{\phi}=1 \otimes h$. Clearly, $\phi$ is an isomorphism of $F$ into $R$. Further- 
more, $h^{\phi}=h$ if and only if $1 \otimes h=h \otimes 1=h$, which is equivalent to $h \in C$. In other words, $C$ is the invariant field of $\phi$. All semi-linear transformations considered henceforth will be $\phi$-semi-linear transformations.

The main object of the present section is to show:

Theorem 2.1. A regular semi-linear transformation $T \in \mathcal{L}$ represents a central simple algebra if and only if the relatively cyclic bimodule $(\mathcal{R}, T)$ is closed.

We begin with some lemmas.

Lemma 2.1. Let $\left\{h_{i}\right\}$ be a base of $F$ over $C$, then the set $\left\{h_{i}^{\phi}\right\}$ is both a right and a left base of $\Re(V)$ over $R\left(V_{F}\right)$.

This lemma is an immediate consequence of the following relation:

$$
\Re(V)=\Re \otimes_{F} \Re\left(V_{F}\right)=\left(F \otimes_{C} F\right) \otimes_{F} \Re\left(V_{F}\right)=F^{\phi} \otimes_{C} \Re\left(V_{F}\right) .
$$

Lemma 2.2. If $T$ is a regular semi-linear transformation of $V_{F}$ into $V$ then $(\mathfrak{R}, T)$ is a regular relatively cyclic $\Re\left(V_{F}\right)$-bimodule.

For, let $P \in \Re\left(V_{F}\right)$ such that $T P=0$, then $T P v=0$ for all $v \in V_{F}$. Since $T$ is regular, it follows that $P v=0$, i.e., $P=0$, which proves (2.1). To prove (2.2), we observe that Lemma 1.3 implies that any semi-linear transformation $S \in R$ has the form $S=P T$ with $P \in \Re(V)$ uniquely determined. By the previous lemma, we have: $P=\sum P_{j} h_{j}^{\phi}, P_{j} \in \Re\left(V_{F}\right)$. Hence, $S=\sum P_{j} h_{j}^{\phi} T=\sum P_{j} T h_{j}$. Furthermore, this representation is unique, since if $\sum P_{j} T h_{j}=0$, then $\left(\sum P_{j} h_{j}^{\phi}\right) T=0$; but $T$ is regular, hence $\sum P_{j} h_{j}^{\phi}=0$, which yields $P_{j}=0$. This proves that $\mathfrak{R}=\Re\left(V_{F}\right) T \Re\left(V_{F}\right)=\Re\left(V_{F}\right) T F$ and that ( $(, T)$ satisfies (2.2).

In particular, the preceding proof shows that

Corollary 2.1. If $T$ is regular and the set $\left\{h_{i}\right\}$ is a base of $F$ over $C$ then $\left\{\boldsymbol{T} h_{i}\right\}$ is a left base of $\mathfrak{Q}$ over $\mathfrak{R}\left(V_{\boldsymbol{F}}\right)$.

We are now in a position to prove Theorem 2.1. The proof follows very closely Nakayama's proof of [12, Proposition 10].

For any $P \in \Re\left(V_{F}\right)$, we have by the previous corollary that $T P=\sum P_{j} T h_{j}$, $P_{j} \in \Re\left(V_{F}\right)$. We set: $f_{j}(P)=P_{j}$. Thus, $f_{j}$ is an additive endomorphism of $\Re\left(V_{F}\right)$ into itself.

Let $(\mathfrak{R}, T)$ be closed, then there exists a homomorphism $\rho:(\mathfrak{R}, T) \rightarrow(\mathfrak{R} \otimes \mathfrak{R}$, $T \otimes T)$ such that $\rho(T)=T \otimes T$. We recall that the Kronecker product $\& \otimes \&$ is taken with respect to $\Re\left(V_{F}\right)$ so that $S_{1} P \otimes S_{2}=S_{1} \otimes P S_{2}$ for $S_{i} \in \mathbb{R}$ and $P \in \Re\left(V_{F}\right)$.

Let $P \in \Re\left(V_{F}\right)$ then $T P=\sum f_{j}(P) T h_{j}$ yields:

$$
\begin{aligned}
\rho(T P) & =\rho(T) P=(T \otimes T) P=T \otimes T P \\
& =T \otimes \sum f_{j}(P) T h_{j}=\sum_{j} T f_{j}(P) \otimes T h_{j} \\
& =\sum_{i, j} f_{i}\left(f_{j}(P)\right) T h_{i} \otimes T h_{j} .
\end{aligned}
$$


On the other hand:

$$
\begin{aligned}
\rho(T P) & =\rho\left(\sum f_{j}(P) T h_{j}\right)=\sum f_{j}(P) \rho(T) h_{j} \\
& =\sum f_{j}(P) T \otimes T h_{j}=\sum_{j, i} f_{j}(P) f_{i}(1) T h_{i} \otimes T h_{j} .
\end{aligned}
$$

Clearly, $T h_{i} \otimes T h_{j}$ form a left base of $\mathfrak{R} \otimes \mathfrak{R}$ over $\Re\left(V_{F}\right)$; hence it follows from (2.3) and (2.4) that

$$
f_{i}\left(f_{j}(P)\right)=f_{j}(P) f_{i}(1) \text { for all } i, j .
$$

From this we conclude that:

$$
T f_{j}(P)=\sum_{i} f_{i}\left(f_{j}(P)\right) T h_{i}=\sum_{i} f_{j}(P) f_{i}(1) T h_{i}=f_{j}(P) T .
$$

Thus, we have: $f_{j}(P)^{T}=f_{j}(P)$, i.e., $f_{j}(P) \in O(T)$.

Furthermore, for every $P \in \Re\left(V_{F}\right): T P=\sum f_{j}(P) T h_{j}=T \sum f_{j}(P) h_{j}$. Hence, the regularity of $T$ implies that $P=\sum f_{j}(P) h_{j}$. Note also that the set $\left\{h_{j}\right\}$ is also independent over $O(T)$, for if $\sum A_{j} h_{j}=0, A_{j} \in O(T)$ then $T \sum A_{j} h_{j}$ $=\sum A_{j} T h_{j}=O$. But $\left\{T h_{j}\right\}$ is a base over $\Re\left(V_{F}\right)$, hence $A_{j}=0$.

From this it follows that $O(T) F \cong O(T) \otimes_{C} F$ and that $O(T) F=\Re\left(V_{\boldsymbol{F}}\right)$. This implies that $O(T)$ is a central simple algebra of order $n^{2}$ over $C$ which is split by $F$; i.e., $T$ represents the central simple algebra $O(T)$.

Conversely, let $T$ represent a central simple algebra $\mathfrak{A}$ and we may assume that $\mathfrak{A}=O(T) \subseteq \mathfrak{R}(V)$. Since $F$ belongs to the centralizer of $\mathfrak{A}$ in $\mathfrak{R}(V)$ and $F$ splits $\mathfrak{A}$, it follows that $\mathfrak{A} \otimes_{C} F=\mathfrak{R}\left(V_{F}\right)$. Hence, $\mathfrak{N}(V)=\mathfrak{A} \otimes R=(\mathfrak{A} \otimes F)$ $\otimes F^{\phi}=\Re\left(V_{F}\right) \otimes F^{\phi}$. This yields readily that $\Re(V) \cong \Re\left(V_{F}\right) \otimes{ }_{A} \Re\left(V_{F}\right)$, since $\Re\left(V_{F}\right) \cong \Re\left(V_{F^{\phi}}\right)=\mathfrak{A} \otimes F^{\phi}$ and, therefore, $\quad \Re\left(V_{F}\right) \otimes_{A} \Re\left(V_{F^{\phi}}\right) \cong \Re\left(V_{F}\right) \otimes F^{\phi}$ $=\Re(V)$.

We also have: $(\mathfrak{R}, \boldsymbol{T})=\left(\Re\left(V_{F}\right) \boldsymbol{T F}, \boldsymbol{T}\right)$ and this bimodule is isomorphic with $\Re\left(V_{F}\right) \otimes_{A} \Re\left(V_{F}\right)$ by the correspondence generated by mapping: $T \rightarrow 1 \otimes 1$. Since $\mathfrak{A}=O(T)$ is the set of all elements which commute with $T$, it follows that $\mathfrak{R}=\mathfrak{R}\left(V_{F}\right) \boldsymbol{T}(\mathfrak{A} \otimes F)=\mathfrak{R}\left(V_{F}\right) T \mathfrak{T}\left(V_{F}\right)$. Clearly, this isomorphism is an $\Re\left(V_{F}\right)-\Re\left(V_{F}\right)$ isomorphism. Hence, the relatively cyclic bimodules $(\mathcal{R}, T)$ and $\left(\Re\left(V_{F}\right) \otimes_{A} \Re\left(V_{F}\right), 1 \otimes 1\right)$ are isomorphic. The latter is known to be closed (e.g., by [12, Proposition 9] and [12, Main Theorem]); hence $(\mathbb{R}, \boldsymbol{T})$ is also closed.

3. Closure of $(\Omega, T)$. We recall that $(\ell, T)$ is closed if there exists an $\Re\left(V_{F}\right)-\Re\left(V_{F}\right)$ homomorphism of $(\mathfrak{R}, T)$ into $(\mathbb{R} \otimes R, T \otimes T)$ which maps $T$ onto $T \otimes T$. In the present section we provide a necessary and sufficient condition for the closure of $(\mathfrak{R}, T)$.

To this end we consider the triple product $F^{3}=F \otimes_{C} F \otimes_{C} F$. As in the preceding section we assume that $F \subset F^{2} \subset F^{3}$ by identifying $F$ with $F \otimes 1$ $=\{h \otimes 1, h \in F\}$ and by identifying the set $F^{2}$ with $F^{2} \otimes 1$. We shall deal with the following isomorphisms: 


$$
\begin{array}{ll}
\phi: F \rightarrow 1 \otimes F ; & h^{\phi}=1 \otimes h \text { for every } h \in F, \\
\psi: F \rightarrow 1 \otimes 1 \otimes F ; & h^{\psi}=1 \otimes 1 \otimes h \text { for } h \in F
\end{array}
$$

and the isomorphisms:

$\alpha: F \otimes F \rightarrow 1 \otimes F \otimes F$ defined by the $(h \otimes k)^{\alpha}=1 \otimes h \otimes k=h^{\phi} k^{\psi}$, $\beta: F \otimes F \rightarrow F \otimes 1 \otimes F$ defined by $(h \otimes k)^{\beta}=h \otimes 1 \otimes k=h k^{\psi}$.

Note that $\alpha \phi=\beta \phi=\psi$ and that the restriction of $\alpha$ to $F$ is $\phi$.

Let $V_{0}$ be a vector space of dimension $n$ over the field $C$. We shall need the following spaces and modules:

(i) $V_{F}=F \otimes_{C} V_{0}$,

(3.3) (ii) $V_{F^{2}}=F^{2} \otimes_{C} V_{0}=F^{2} \otimes_{F} V_{F}=F^{\phi} \otimes_{C} V_{F}$,

(iii) $V_{F^{3}}=F^{3} \otimes_{C} V_{0}=F^{3} \otimes_{F} V_{F}=F^{3} \otimes_{F^{2}} V_{F^{2}}$.

For $v \in V_{0}$ and $r \in F^{3}$ we shall set $r v=r \otimes v$. Similarly we put $h \otimes k \otimes l \otimes v$ $=h k^{\phi} l^{\psi} v$ for $h, k, l \in F$.

Each of the relations of (3.3) yield the following relations between the respective rings of endomorphisms:

(i) $\Re\left(V_{F}\right)=F \otimes_{C} \Re\left(V_{0}\right)$,

(ii) $\Re\left(V_{F^{2}}\right)=F^{2} \otimes_{C} \Re\left(V_{0}\right)=F^{2} \otimes_{F} \Re\left(V_{F}\right)$,

(iii) $\Re\left(V_{F^{3}}\right)=F^{3} \otimes_{C} \Re\left(V_{0}\right)=F^{3} \otimes_{F} \Re\left(V_{F}\right)=F^{3} \otimes_{F^{2}} \Re\left(V_{F^{2}}\right)$.

Since $F^{2}=F F^{\phi}$ and $F^{3}=F F^{\phi} F^{\psi}=F^{2} F^{\psi}$ it follows by (3.4 ii) and (3.4 iii) that

(i) $\Re\left(V_{F^{2}}\right)=F^{\phi} \otimes_{C} \Re\left(V_{F}\right)$,

(ii) $\Re\left(V_{F^{3}}\right)=F^{\phi} F^{\psi} \otimes_{C} \Re\left(V_{F}\right)=F^{\phi \alpha} \otimes_{C} \Re\left(V_{F}\right)=F^{\psi} \otimes_{C} \Re\left(V_{F^{2}}\right)$.

With each of the isomorphisms of (3.1) and (3.2) we associate the following four bimodules of semi-linear transformations:

$\mathfrak{l}=\mathfrak{R}_{\phi}$ : the $\Re\left(V_{F}\right)$ bimodule of all $\phi$-semi-linear transformations of $V_{F}$ into $V_{F^{2}}$.

$\mathfrak{R}_{\psi}$ : the $\Re\left(V_{F}\right)$ bimodule of all $\psi$-semi-linear transformations of $V_{F}$ into $V_{F^{3}}$.

$\mathfrak{R}_{\alpha}$ : the $\Re\left(V_{F^{2}}\right)$ bimodule of all $\alpha$-semi-linear transformations of $V_{F^{2}}$ into $V_{F^{3}}$.

$\mathfrak{R}_{\beta}$ : the $\Re\left(V_{F^{2}}\right)$ bimodule of all $\beta$-semi-linear transformations of $V_{F^{2}}$ into $V_{F^{3}}$.

Each of the bimodules $\mathfrak{R}_{\gamma}, \gamma=\phi, \psi, \alpha, \beta$ contains a regular $\gamma$-semi-linear transformation $E_{\gamma}$ which is the unique extension of the injection of $V_{0}$ into the respective space $V_{F}{ }^{i}$. Namely, if $v_{1}, \cdots, v_{n}$ is a $C$-base of $V_{0}$ then

$$
E_{\gamma}\left(\sum r_{i} v_{i}\right)=\sum r_{i}^{\gamma} v_{i}
$$


For further reference we observe that any $\gamma$-semi-linear transformation is uniquely determined by its effect on $V_{0}$.

Lemma 3.1. Let $T$ be a regular $\gamma$-semi-linear transformation and $\left\{h_{i}\right\}$ be a $C$-base of $F$, then

(1) for $\gamma=\phi$, the set $\left\{T h_{i}\right\}$ is a left $\Re\left(V_{F}\right)$-base of $\mathbb{R}_{\phi}$;

(2) for $\gamma=\psi$, the set $\left\{h_{i}^{\phi} T h_{j}\right\}$ is a left $\Re\left(V_{F}\right)$-base of $\mathbb{R}_{\psi}$;

(3) for $\gamma=\alpha$, the set $\left\{T h_{i} h_{j}^{\phi}\right\}$ is a left $\Re\left(V_{F}\right)$-base of $\mathfrak{R}_{\alpha}$.

Proof. It follows by Lemma 1.3 that $\mathfrak{\ell}_{\phi}=\Re\left(V_{F^{2}}\right) \boldsymbol{T}=\left[\Re\left(V_{F}\right) \otimes_{C} F^{\phi}\right] \boldsymbol{T}$ $=\Re\left(V_{F}\right) T \otimes F=\Re\left(V_{F}\right) T F$ from which one readily derives (1). Similarly (2) and (3) follows from the following relations:

$$
\begin{aligned}
& \mathfrak{\imath}_{\psi}=\Re\left(V_{F^{3}}\right) T=\left[\Re\left(V_{F}\right) \otimes_{C} F^{\phi} F^{\psi}\right] T=\Re\left(V_{F}\right) \otimes_{C} F^{\phi} T F . \\
& \mathfrak{R}_{\alpha}=\Re\left(V_{F^{3}}\right) T=\left[\Re\left(V_{F}\right) \otimes_{C}\left(F^{2}\right)^{\alpha}\right] T=\Re\left(V_{F}\right) \otimes T F^{2}=\Re\left(V_{F}\right) T F^{2} .
\end{aligned}
$$

We shall need also the following simple properties of the transformations $E_{\gamma}:$

(i) $E_{\alpha} E_{\phi}=E_{\psi}$.

(ii) $\Re\left(V_{0}\right) \subseteq O\left(E_{\gamma}\right)$ for $\gamma=\phi$, $\alpha$; i.e., $P E_{\gamma}=E_{\gamma} P$ for $P \in \Re\left(V_{0}\right)$.

(iii) $\Re\left(V_{F}\right) \subseteq O\left(E_{\gamma}\right)$ for $\gamma=\psi, \beta$.

Let $\boldsymbol{S} \in \mathfrak{R}_{\phi}$, then the mapping $\boldsymbol{S}^{\alpha}=\alpha \otimes S: V_{F^{2}} \rightarrow V_{F^{3}}$ defined by

$$
S^{\alpha}(r \otimes v)=(\alpha \otimes S)(r \otimes v)=r^{\alpha} S v, r \in F^{2} \text { and } v \in V_{F}
$$

is a well defined $\alpha$-semi-linear transformation of $V_{F^{2}}$ into $V_{F^{3}}$. Actually, $S$ is uniquely determined by the property that its restriction to $V_{F}$ is $S$. Indeed, if there exists a unique $\alpha$-semi-linear transformation $S^{\prime}$ which has the same effect on $V_{0}$ as $S$, then since the restriction of $\alpha$ to $F$ is $\phi$, it follows immediately that $S^{\prime}$ and $S$ have also the same effect on $V_{F}$; hence, clearly $S^{\prime}$ is the $\alpha$-semi-linear transformation $S^{\alpha}$ which satisfies (3.8).

It follows readily from the last property of $S$ that:

Lemma 3.2. The mapping $\boldsymbol{S} \rightarrow \boldsymbol{S}^{\alpha}$ is an $\Re\left(V_{F}\right)-\mathfrak{R}\left(V_{F}\right)$ isomorphism of $\mathfrak{R}_{\phi}$ into $\Omega_{\alpha}$.

Consider now the product $\ell_{\phi} \otimes_{R\left(V_{F}\right)} \mathfrak{\ell}_{\phi}$. We show:

Lemma 3.3. The mapping $\rho: S \otimes T \rightarrow S^{\alpha} T, S, T \in \Omega_{\phi}$ determines an $R\left(V_{F}\right)$ $-R\left(V_{F}\right)$ isomorphism of $\mathfrak{R}_{\phi} \otimes \mathfrak{R}_{\phi}$ onto $\mathfrak{R}_{\psi}$.

Proof. Since $T: V_{F} \rightarrow V_{F^{2}}$ and $S: V_{F^{2}} \rightarrow V_{F^{3}}$, it follows that $S^{\alpha} T$ is a well defined homomorphism of $V_{F}$ into $V_{F^{3}}$. Furthermore, $S^{\alpha} T \in \mathbb{R}_{\psi}$, since

$$
S^{\alpha} T h v=S^{\alpha} h^{\phi} T v=h^{\alpha \phi} S^{\alpha} T v=h^{\psi} S^{\alpha} T v \text { for } h \in F \text { and } v \in V_{F} \text {. }
$$

The mapping $\rho$ is defined as $\rho\left(\sum S_{i} \otimes T_{i}\right)=\sum S_{i}^{\alpha} T_{i}$, and to prove that $\rho$ 
is a well defined additive homomorphism of $\mathbb{R}_{\phi} \otimes \mathbb{R}_{\phi}$ into $\mathfrak{R}_{\psi}$, it suffices to show that if a relation $\sum S_{i} \otimes T_{i}=0$ holds in $\mathfrak{R}_{\phi} \otimes \Omega_{\phi}$ then the $\rho$-image of this element in $\Re_{\psi}$ is also zero. Indeed, let $\sum S_{i} \otimes T_{i}=0$, then it follows, by Lemma 3.1 , that $T_{i}=\sum_{k} P_{i k} E_{\phi} h_{k}, P_{i k} \in R\left(V_{F}\right)$ and where $\left\{h_{k}\right\}$ is a base of $F$ over $C$ and $E_{\phi}$ is the $\phi$-semi-linear transformation defined in (3.6). Thus:

$$
\sum_{i} S_{i} \otimes T_{i}=\sum_{i k} S_{i} \otimes P_{i k} E_{\phi} h_{k}=\sum_{k}\left(\sum_{i} S_{i} P_{i k}\right) \otimes E_{\phi} h_{k}=0
$$

since the tensor product is taken with respect to $\Re\left(V_{F}\right)$. Again, by Lemma 3.1 it follows that $\left\{E_{\phi} h_{k}\right\}$ is an $\Re\left(V_{F}\right)$-base of $\mathfrak{\Omega}_{\phi}$; hence, it follows by (3.9) that $\sum_{i} S_{i} P_{i k}=0$ for all $k$.

Consequently it follows by Lemma 3.2 that:

$$
\rho\left(\sum S_{i} \otimes T_{i}\right)=\sum S_{i}^{\alpha} T_{i}=\sum_{i k} S_{i}^{\alpha} P_{i k} E_{\phi} h_{k}=\sum_{k}\left(\sum_{i} S_{i} P_{i k}\right)^{\alpha} E_{\phi} h_{k}=0
$$

which proves that $\rho\left(\sum S_{i} \otimes T_{i}\right)=0$ if $\sum S_{i} \otimes T_{i}=0$.

The map $\rho$ preserves also right and left multiplications by elements of $\Re\left(V_{F}\right)$. Indeed, for $P \in \Re\left(V_{F}\right), S$ and $T \in \Omega_{\phi}$, we have:

$$
\begin{aligned}
& \rho[(S \otimes T) P]=\rho(S \otimes T P)=S^{\alpha}(T P)=\left(S^{\alpha} T\right) P=[\rho(S \otimes T)] P, \\
& \rho[P(S \otimes T)]=\rho(P S \otimes T)=(P S)^{\alpha} T=P\left(S^{\alpha} T\right)=P[\rho(S \otimes T)] .
\end{aligned}
$$

To prove that $\rho$ is an isomorphism, it suffices to show that $\rho$ induces a one-to-one correspondence between a left $\Re\left(V_{F}\right)$-base of $\mathfrak{R}_{\phi} \otimes \mathfrak{R}_{\phi}$ and a left $\Re\left(V_{F}\right)$-base of $\AA_{\psi}$.

From Lemma 3.1 we know that $\left\{E_{\phi} h_{i}\right\}$ is a left $\Re\left(V_{F}\right)$-base of $\mathfrak{R}_{\phi}$; hence, since $\Re\left(V_{F}\right)$ is a finite dimensional simple ring, it follows that the set $\left\{E_{\phi} h_{i} \otimes E_{\phi} h_{j}\right\}$ is a left $\Re\left(V_{F}\right)$-base of $\mathfrak{R}_{\phi} \otimes \mathbb{R}_{\phi}$.

For every $v \in V_{0}, E_{\phi}^{\alpha} E_{\phi} v=E_{\psi} v=v$ which implies that both $E_{\phi}^{\alpha} E_{\phi}$ and $E_{\psi}$ have the same effect on $V_{0}$. Hence, $E_{\psi}=E_{\phi}^{\alpha} E_{\phi}$. It follows now that:

$$
\rho\left(E_{\phi} h_{i} \otimes E_{\phi} h_{j}\right)=\left(E_{\phi} h_{i}\right)^{\alpha}\left(E_{\phi} h_{j}\right)=h_{i}^{\phi} E_{\phi}^{\alpha} E_{\phi} h_{j}=h_{i}^{\phi} E_{\psi} h_{j},
$$

and from Lemma 3.2 it follows that the set $\left\{h_{i}^{\phi} E_{\psi} h_{j}\right\}$ is a left $\Re\left(V_{F}\right)$-base of $\mathfrak{R}_{\psi}$. This completes the proof of the lemma.

We turn now to a different method of turning $\phi$-semi-linear transformations into $\psi$-semi-linear transformations:

Lemma 3.4. If $T \in R_{\phi}$ then $T^{\beta}=E_{\beta} T$ is a $\psi$-semi-linear transformation of $V_{F}$ into $V_{F^{3}}$; and the mapping $\sigma: T \rightarrow T^{\beta}$ is an $\Re\left(V_{F}\right)-\Re\left(V_{F}\right)$ isomorphism of $\Re_{\phi}$ into $\Re_{\psi}$.

Proof. Since $T: V_{F} \rightarrow V_{F^{2}}$ and $E_{\beta}: V_{F^{2}} \rightarrow V_{F^{3}}$ it follows that $E^{\beta} T$ is a well defined homomorphism of $V_{F}$ into $V_{F^{3}}$. Moreover, for $h \in F$ and $v \in V_{F}$ we have 


$$
T^{\beta}(h v)=E_{\beta} T(h v)=E_{\beta} h^{\phi} T v=h^{\beta \phi} E_{\beta} T v=h^{\psi} T^{\beta} v
$$

since $\phi=\beta \psi$. This proves that $T \in \mathfrak{R}_{\psi}$.

Clearly, $(T+S)^{\beta}=T^{\beta}+S^{\beta}$ and $(T P)^{\beta}=T^{\beta} P$ for $P \in \Re\left(V_{F}\right)$. To prove that $(P T)^{\beta}=P T^{\beta}$ we apply $(3.7 \mathrm{iii})$ and we obtain that $(P T)^{\beta}=E_{\beta} P T=P E_{\beta} T$ $=P T^{\beta}$.

One readily verifies by the regularity of $E_{\beta}$ that $T^{\beta}=0$ if and only if $T=0$, and the proof of the lemma is completed.

We are now in position to give a criterion for the closure of the relatively cyclic bimodule $(\&, T)$.

The main result of the present section is the following:

THEOREM 3.1. Let $T$ be a $\phi$-regular semi-linear transformation of $V_{F}$ into $V_{F^{2}}$, then $\left(L_{\phi}, T\right)$ is closed if and only if $T$ satisfies the condition:

$$
T^{\alpha} T=t T^{\beta} \text { for some regular element } t \in F^{3} .
$$

Proof. $T$ is closed if and only if there exists an $\Re\left(V_{F}\right)-\Re\left(V_{F}\right)$ homomorphism $\delta:\left(\Omega_{\phi}, T\right) \rightarrow\left(\Omega_{\phi} \otimes \Omega_{\phi}, T \otimes T\right), \delta(T)=T \otimes T$. Now, Lemma 3.4 yields an $\Re\left(V_{F}\right)-\Re\left(V_{F}\right)$ isomorphism $\sigma$ of $\mathfrak{R}_{\phi}$ into $\mathfrak{R}_{\psi}$. Put $\sigma\left(\mathfrak{R}_{\phi}\right)=\mathfrak{R}_{\phi}^{\beta}=\left\{T^{\beta} ; T \in \mathbb{R}_{\phi}\right\}$ $\subseteq \Omega_{\psi}$. In Lemma 3.3 we have another $\Re\left(V_{F}\right)-\Re\left(V_{F}\right)$ isomorphism $\rho:\left(\Omega_{\phi} \otimes \Omega_{\phi}, T \otimes T\right) \rightarrow\left(\Omega_{\psi}, T^{\alpha} T\right)$. Thus, the problem of the closure of $\left(\Omega_{\phi}, T\right)$ reduces to the problem of determining an $\Re\left(V_{F}\right)-\Re\left(V_{F}\right)$ homomorphism $\tau:\left(\ell_{\phi}^{\beta}, T^{\beta}\right) \rightarrow\left(\ell_{\psi}, T^{\alpha} T\right)$ such that $\tau\left(T^{\beta}\right)=T^{\alpha} T$.

Our theorem will follow by showing that the $\Re\left(V_{F}\right)-\Re\left(V_{F}\right)$ homomorphisms of $\mathfrak{R}_{\phi}^{\beta}$ into $\mathfrak{R}_{\psi}$ are left multiplications by elements $t \in F^{3}$.

Indeed, let $\tau: \mathfrak{R}_{\phi}^{\beta} \rightarrow \mathfrak{R}_{\psi}$ be an $\Re\left(V_{F}\right)-\Re\left(V_{F}\right)$ homomorphism. Let $\left\{v_{i}\right\}$ be the fixed base of $V_{0}$. Consider the linear transformations $E_{i k} \in \Re\left(V_{F^{3}}\right)$ defined by: $E_{i k} v_{j}=v_{i} \delta_{k j}$ where $\delta_{k j}$ is the Kronecker $\delta$. One readily verifies, by standard methods, that the linear transformations of $V_{F^{3}}$ which commute with all $E_{i k}$ are the elements $t \in F^{3}$, i.e., the linear transformations: $v \rightarrow t v$, $v \in V$. Note also that $E_{i k} \in \Re\left(V_{0}\right)$, hence $E_{i k} E_{\gamma}=E_{\gamma} E_{i k}$ for $\gamma=\phi, \psi, \alpha$ and $\beta$.

First we observe that $\sigma\left(E_{\phi}\right)=E_{\beta} E_{\phi}=E_{\psi}$, since both are $\psi$-semi-linear transformation with the same effect on $V_{0}$. From Lemma 1.3 it follows that $\mathfrak{R}_{\psi}=\Re\left(V_{F^{s}}\right) E_{\psi}$; hence, since $\tau\left(E_{\psi}\right) \in \mathfrak{R}_{\psi}$, we have: $\tau\left(E_{\psi}\right)=P E_{\psi}$, where $P \in \Re\left(V_{F^{3}}\right)$. Now $\tau$ is an $\Re\left(V_{F}\right)-\Re\left(V_{F}\right)$ homomorphism; hence:

$$
\tau\left(E_{\psi} E_{i k}\right)=\tau\left(E_{\psi}\right) E_{i k}=P E_{\psi} E_{i k}=P E_{i k} E_{\psi} .
$$

On the other hand,

$$
\tau\left(E_{i k} E_{\psi}\right)=E_{i k} \tau\left(E_{\psi}\right)=E_{i k} P E_{\psi} .
$$

Thus, the relation $E_{i k} E_{\psi}=E_{\psi} E_{i k}$ yields $\left(E_{i k} P-P E_{i k}\right) E_{\psi}=0$. Since $E_{\psi}$ is a regular $\psi$-linear transformation, it follows that $E_{i k} P=P E_{i k}$ for all $i, k$. Hence, $P=t \in F^{3}$.

The general $\psi$-semi-linear transformation $S^{\beta} \in \mathbb{R}_{\phi}^{\beta}$ has, in view of Lemma 
3.1 , the form $S^{\beta}=\left(\sum P_{i} E_{\phi} h_{i}\right)^{\beta}=\sum P_{i} E_{\phi}^{\beta} h_{i}=\sum P_{i} E_{\psi} h_{i}$, where $P_{i} \in \Re\left(V_{F}\right)$ and $\left\{h_{i}\right\}$ is a base of $F$ over $C$. Hence,

$$
\tau(S)=\sum P_{i} \tau\left(E_{\psi}\right) h_{i}=\sum P_{i} t E_{\psi} h_{i}=t \sum P_{i} E_{\psi} h_{i}=t S .
$$

This shows that $\tau$ is obtained by multiplying the elements of $\mathfrak{R}_{\phi}^{\beta}$ on the left by an element $t \in F^{3}$.

The converse, namely that the mapping $S^{\beta} \rightarrow t S^{\beta}$, for some fixed $t \in F^{3}$, is an $\Re\left(V_{F}\right)-\Re\left(V_{F}\right)$ homomorphism of $\mathfrak{R}_{\phi}^{\beta}$ in to $\mathfrak{R}_{\psi}$ is readily verified.

From the result obtained so far, we see that $\left(\Omega_{\phi}, T\right)$ is closed if and only if $\tau\left(T^{\beta}\right)=T^{\alpha} T$ for some homomorphism $\tau: \mathbb{R}_{\phi}^{\beta} \rightarrow \mathfrak{R}_{\psi}$-and this has been shown to be equivalent to the fact that $T^{\alpha} T=t T^{\beta}$ for some $t \in F^{3}$; i.e., condition (3.10).

It remains only to show that $t$ is necessarily a regular element in $F^{3}$. Indeed, it is not difficult to show that the regularity of $T$ implies that $T^{\alpha} T$ is also a regular $\psi$-semi-linear transformation, and so is $T^{\beta}$. It follows now immediately by Lemma 1.3 that $t$ is a regular linear transformation in $\Re(V)$, which is equivalent to the fact that $t$ is regular in $F^{3}$.

We wish to replace condition (3.10) by a more applicable condition on linear transformation. To this end we observe that (3.4) imply that the isomorphisms $\phi, \alpha$ and $\beta$ can be extended uniquely to isomorphism of $\Re\left(V_{F}\right)$ and $\Re\left(V_{F^{2}}\right)$, respectively, which will leave the elements of $\Re\left(V_{0}\right)$ invariant. We shall use the same notations $\phi, \alpha$ and $\beta$ for the extended isomorphisms. More precisely, if $\left\{h_{i}\right\}$ is a $C$-base of $F$ then every $P \in \Re\left(V_{F}\right)$ can be written uniquely (in view of $(3.4 \mathrm{i})$ ) in the form $P=\sum h_{i} P_{i}^{0}, P_{i}^{0} \in \Re\left(V_{0}\right)$, then

$$
\text { (i) } P^{\phi}=\sum h_{i}^{\phi} P_{i}^{0}
$$

and $\phi$ is an isomorphism of $\Re\left(V_{F}\right)$ in to $\Re\left(V_{F^{2}}\right)$. Similarly for $P \in \Re\left(V_{F^{2}}\right)$ then $P=\sum h_{i} h_{j}^{\phi} P_{i j}^{0}, P_{i j}^{0} \in \Re\left(V_{0}\right)$, by (3.4ii), and we have

$$
\begin{aligned}
& \text { (ii) } P^{\alpha}=\sum h_{i}^{\phi} h_{j}^{\beta} P_{i j}^{0}, \\
& \text { (iii) } P^{\beta}=\sum h_{i} h_{j}^{\psi} P_{i j}^{0} .
\end{aligned}
$$

Our new criterion for the closure of $(\mathcal{R}, T)$ is the following:

Theorem 3.2. Let $P$ be a regular linear transformation of $V_{12}$, then the $\phi$-semi-linear transformation $T=P E_{\phi}$ represents a simple algebra if and only if $P$ satisfies the condition;

$$
P P^{\alpha}=t P^{\beta} \text { for some regular } t \in F^{3} \text {. }
$$

Proof. It follows readily by $(3.7 \mathrm{ii})$ and (3.11) that $E_{\gamma} P=P^{\gamma} E_{\gamma}$ for $\gamma$ $=\phi, \alpha, \beta$. Hence:

$$
T^{\alpha} T=\left(P E_{\phi}\right)^{\alpha} P E_{\phi}=P E_{\phi}^{\alpha} P E_{\phi}=P P^{\alpha} E_{\phi}^{\alpha} E_{\phi}=P P^{\alpha} E_{\psi} .
$$


On the other hand, it follows by (3.4) that

$$
T^{\beta}=E_{\beta}\left(P E_{\phi}\right)=P^{\beta} E_{\beta} E_{\phi}=P^{\beta} E_{\psi} .
$$

Since one readily verifies that $E_{\phi}^{\alpha}=E_{\alpha}$, as two $\alpha$-semi-linear transformations with the same effect on $V_{0}$, and the same reason yields that $E_{\phi}^{\alpha} E_{\phi}$ $=E_{\beta} E_{\phi}=E_{\psi}$. The regularity of $E_{\psi}$ implies now immediately that (3.9) and the condition of our theorem are equivalent.

For further applications we need also a condition for a linear transformation $P$ satisfying (3.12) to represent the complete matrix ring $C_{n}$.

Theorem 3.3. For a regular tinear transformation $P \in \Re\left(V_{F^{2}}\right)$, the $\phi$ semi-linear transformation $P E_{\phi}$ represents the matrix ring, if and only if:

$$
P=r Q^{-1} Q^{\phi} \text { for some regular } r \in F^{2} \text { and } Q \in \Re\left(V_{F}\right) \text {. }
$$

Proof. One readily verifies that $O\left(E_{\phi}\right) \cong C_{n}$; i.e., $E_{\phi}$ represents $C_{n}$. If $P E_{\phi}$ represents $C_{n}$ then $O\left(E_{\phi}\right) \cong O\left(P E_{\phi}\right) \cong C_{n}$; hence, Corollary 1.2 yields that $P E_{\phi}=r Q^{-1} E_{\phi} Q=r Q^{-1} Q^{\phi} E_{\phi}$, which is equivalent to $P=r Q^{-1} Q^{\phi}$. The converse follows by the same corollary.

More generally, Corollary 1.2 yields in a similar way:

THeOREM 3.4. Two $\phi$-semi-linear transformations $P_{1} E_{\phi}$ and $P_{2} E_{\phi}$ represent isomorphic algebras, if and only if:

$$
P_{1}=r Q^{-1} P_{2} Q^{\phi} \text { for some regular } r \in F^{2}, Q \in \Re\left(V_{F}\right) .
$$

4. The space $F \otimes K$. Let $K$ be an algebraic extension of $C$ of degree $n$. We consider $K$ as a $C$-vector space of dimension $n$ and we apply the result of the preceding section with the space $V_{0}=K$. Since $K$ is a ring, the spaces $K_{F}=F \otimes K, K_{F^{2}}=F^{2} \otimes K$ and $K_{F^{3}}=F^{3} \otimes K$ are also associative rings $\left({ }^{7}\right)$ and we shall use this additional property to obtain a special form of the linear transformations $P$ which satisfy (3.12). The multiplication in the ring $K_{F}$ will then be used to obtain a representation of the Brauer group of algebras which are split both by $F$ and $K$. The context of the present section follows closely the result obtained in $[2, \S 3]$, which covers a special case of the results obtained here.

We shall identify the element $1 \otimes k, k \in K$, with the element $k$ and we shall write $r k=r \otimes k$ for $r \in F^{3}$ and $k \in K$.

For a fixed $a \in K_{F}$ the mapping: $m \rightarrow a m, m \in K_{F}$, where the multiplication $a m$ is the multiplication of two elements in the ring $K_{F}$, determines a linear transformation of the space $K_{F}$. In particular, multiplications by elements of the field $K$ is a field isomorphic with $K$. We shall denote this field by $K^{m}$.

Let $\mathfrak{A}$ be a central simple algebra of order $n^{2}$ over $C$ which is split by $F$ and which contains the field $K$ as a maximal commutative field. Since

${ }^{(7)}$ All tensor products which will be used henceforth will be relative to $C$ unless stated otherwise. 
$\left(K_{F} ; F\right)=n$, it follows by Theorem 1.1 that $\mathfrak{A}$ can be represented by a $\phi$ semi-linear transformation $T$ of $K_{F}$ into $K_{F}$, i.e., $\mathfrak{A}=O(T)$. Hence, $O(T)$ contains a field $K_{0}$ isomorphic with $K$. Thus, $K_{0}$ and the field $K^{m}$, which was defined above, are two isomorphic subfields of $\Re\left(K_{F}\right)$. This isomorphism can be extended to an inner isomorphism of $\Re\left(K_{F}\right)$. That is: $K^{m}=P^{-1} K_{0} P$ for some regular $P \in \Re\left(K_{F}\right)$. By considering the $\phi$-semi-linear transformation $T_{0}=P^{-1} T P$, we obtain $O\left(T_{0}\right)=P^{-1} O(T) P$. Hence $O\left(T_{0}\right) \supseteq P^{-1} K_{0} P=K^{m}$. We shall, henceforth, identify the field $K^{m}$ with $K$.

In view of this result, whenever we shall deal, henceforth, with a central simple algebra $\mathfrak{A}$ of order $n^{2}$ over $C$ containing a field isomorphic with $K$, we may assume that $\mathfrak{A}$ is a ring of linear transformations of $K_{F}$ represented by a $\phi$-semi-linear transformation $T$, and that $\mathfrak{A}$ contains the field $K$.

Since the elements of the rings $K, K_{F}, K_{F^{3}}$ appear in the proofs of the present section both as vectors of a space and as linear transformations on the same space, we shall write $T(k)$ or $P(k)$ (instead of $T k, P k$ ) to mean the image of the vector $k$ under the transformation $T$ or $P$; and we shall leave the notations $T k, P k$ for the product of the linear transformation $T$ and $k$, or $P$ and $k$.

Lemma 4.1. Let $T$ be a $\phi$-semi-linear transformation of $K_{F}$ into $K_{F}{ }^{3}$. If $O(T) \supseteq K$, then $T$ is uniquely determined by the element $T(1)=T(1 \otimes 1) \in K_{F}$. Furthermore, $T$ is regular if and only if $T(1)$ is a regular element of $K_{F}$.

Proof. Since $k \in K \subseteq O(T)$, it follows that $k \boldsymbol{T}=\boldsymbol{T} k$. Now $\boldsymbol{T}(k)=\boldsymbol{T}(k \cdot 1)$ $=T k(1)=k T(1)$. Now, $E_{\phi}$ was a $\phi$-semi-linear transformation defined so that $E_{\phi}(k)=k$ for all $k \in V_{0}=K$; hence, both $T(1) E_{\phi}$ and $T$ are $\phi$-semi-linear transformations with the same effect on $K$. Consequently, $T=T(1) E_{\phi}$.

In view of the last lemma, we associate the element $a=T(1) \in K_{F^{2}}$ to any central simple algebra $\mathfrak{A}$ represented in $K_{F}$ by a semi-linear transformation $T$. Thus, we can restate Theorems $3.2,3.3$ and 3.4 in the following way:

THEOREM 4.1. The correspondence $\mathfrak{A} \rightarrow a$ satisfies the following conditions:

(1) $a \in F^{2} \otimes K=K_{F^{2}}$ represents an algebra $A$, if and only if a satisfies:

$$
a a^{\alpha}=t a^{\beta} \text { for some regular element } t \in F^{3} \text {. }
$$

(2) The elements $a, b$ represent isomorphic algebras, if and only if one of them satisfies (4.1) and:

$$
a=r q^{-1} q^{\phi} b \text { for some regular elements } q \in F \otimes K, r \in F^{2} .
$$

(3) The element a represents the matrix ring $C_{n}$, if and only if:

$$
a=r q^{-1} q^{\phi}, q \in F \otimes K \text { and } r \in F^{2} .
$$

Remark 4.1. For further applications, we obtain by (3.1) the following explicit forms of the isomorphisms $\phi, \alpha, \beta$ of $K_{F}$ and $K_{F^{2}}$ : The isomorphism $\phi$ is determined by the relation $(h \otimes k)^{\phi}=1 \otimes h \otimes k=h^{\phi} k, h \in F$ and $k \in K$. Sim- 
ilarly $\alpha$ and $\beta$ are determined by the conditions $\left(h_{1} \otimes h_{2} \otimes k\right)^{\alpha}=1 \otimes h_{1} \otimes h_{2} \otimes k$ $=h_{1}^{\phi} h_{2}^{\psi} k$ and $\left(h_{1} \otimes h_{2} \otimes k\right)^{\beta}=h_{1} \otimes 1 \otimes h_{2} \otimes k=h_{1} h_{2}^{\psi} k$.

Clearly, (4.1) is equivalent to (3.12). To prove that (4.2) is equivalent to (3.14), one has to show that if $T=a E_{\phi}$ and $S=b E_{\phi}$ satisfy the relation $T$ $=r Q^{-1} S Q, r \in F^{2}, Q \in \Re\left(K_{F}\right)$ of Corollary 1.2 -then $Q$ can be taken to be an element $q \in K_{F}$; i.e., the linear transformation $Q$ of $K_{F}$ is multiplication by an element $q$ of $K_{F}$.

Indeed, since $T=r Q^{-1} S Q$, it follows by Corollary 1.1 that $O(T)=Q^{-1} O(S) Q$. Both $O(T)$ and $O(S)$ contain $K$; hence, $O(T)$ contains $K$ and the field $Q^{-1} K Q$. The isomorphism between $K$ and $Q^{-1} K Q$ can be extended to an inner automorphism in $O(T)$, since $O(T)$ is a central simple algebra of finite dimension. Thus $Q^{-1} k Q=P^{-1} k P$ for some $P \in O(T)$. Consequently, $\left(Q P^{-1}\right) k=k\left(Q P^{-1}\right)$ for all $k \in K_{F}$. But $K$ is a maximal field in $\Re\left(K_{F}\right)$, hence it is its own centralizer in $\Re\left(K_{F}\right)$. Consequently, the last relation yields that $Q P^{-1}=q$ for some $q \in K_{F}$. Hence, $Q T Q^{-1}=q\left(P T P^{-1}\right) q^{-1}=q T q^{-1}$, since $P \in O(T)$. This yields $T=r Q^{-1} S Q=r q^{-1} S q$, which proves our assertion.

We follow now the proofs of [2, Theorem 3.1] and [2, Theorem 3.2], and we obtain the following representation of the Brauer group of algebras which are split both by $F$ and $K$.

We recall that two central simple algebras $\mathfrak{A}$ and $\mathfrak{B}$ over $C$ of finite dimension are said to be similar (denoted by $\mathfrak{A} \sim \mathfrak{B}$ ) if $\mathfrak{A}_{m} \cong \mathfrak{B}_{r}$ for some integers $m, r$, where $\mathfrak{A}_{m}$ denotes the complete matrix ring of order $m^{2}$ over $\mathfrak{A}$ and $\mathfrak{B}_{r}$ has a similar meaning. The set of all central simple algebras of finite dimension form a group with respect to the relation of similarity and the Kronecker product $\otimes$. The subset of all central algebras which are split both by $K$ and $F$ form a subgroup, which we shall denote by $\mathfrak{B}(K, F)$. The group of all algebras split by $F$, i.e. the Brauer group of algebras, will be denoted by $\mathfrak{B}(F)$.

Now the set $\mathcal{Z}$ of all regular elements of $F^{2} \otimes K$ which satisfy (4.1) is readily seen to form a multiplicative subgroup of the group of all regular elements of $F^{2}$. The set $B$ of all elements of $F^{2} \otimes K$ satisfying (4.3) is, clearly, a subgroup of $\mathcal{Z}$. The main result of the present section is:

Theorem 4.2. The Brauer group $\mathfrak{B}(K, F)$ is isomorphic with $\mathrm{Z} / \mathfrak{B}$.

We begin with the proof of a lemma which is a generalization of $[1$, Theorem 3.1];

Lemma 4.2. Let $\mathrm{M}: K_{F}{ }^{3} \otimes K_{F^{3}} \rightarrow K_{F^{3}}$ denote the multiplication of $K_{F}$, i.e., $\mathbf{M}\left(a_{1} \otimes a_{2}\right)=a_{1} a_{2} ;$ and let $a E_{\phi}, b E_{\phi}$ be two $\phi$-semi-linear transformations of $K_{F}$ into $K_{F^{3}}$. Then $\mathrm{M}$ satisfies the relation $\mathrm{M}\left(a E_{\phi} \otimes b E_{\phi}\right)=(a b) E_{\phi} \mathrm{M}$.

Indeed, $a E_{\phi} \otimes b E_{\phi}$ is a $\phi$-semi-linear transformation of $K_{F} \otimes K_{F}$ and for $k_{i}, k_{j} \in K_{F}$ we have:

$$
\begin{aligned}
\mathrm{M}\left(a E_{\phi} \otimes b E_{\phi}\right)\left(k_{i} \otimes k_{j}\right) & =\mathrm{M}\left(a E_{\phi}\left(k_{i}\right) \otimes b E_{\phi}\left(k_{j}\right)\right)=\mathrm{M}\left(a k_{i} \otimes b k_{j}\right), \\
& =a k_{i} b k_{j}=(a b)\left(k_{i} k_{j}\right)=(a b) E_{\phi}\left(k_{i} k_{j}\right)=a b E_{\phi} \mathrm{M}\left(k_{i} \otimes k_{j}\right),
\end{aligned}
$$


which clearly yields the lemma.

We turn now to the proof of Theorem 4.2:

If $\mathfrak{A}$ is a central simple algebra split by $K$, then it is well known that $\mathfrak{A}$ is similar to a central simple algebra $\mathfrak{A}_{0}$ of order $n^{2}$ containing $K$ as a maximal field; and all algebras similar to $\mathfrak{A}$ and which are of order $n^{2}$ are isomorphic. Thus, we may assume that the elements of $\mathfrak{B}(K, F)$ are subalgebras of $\Re\left(K_{F}\right)$ and by Theorem 4.1 we may associate an element $a \in F^{2} \otimes K$ with such an algebra.

Conditions (4.1) and (4.2) imply that this mapping is a one-to-one correspondence between $\mathfrak{B}(K, F)$ and $\mathrm{Z} / \boldsymbol{B}$. To prove that this correspondence is an isomorphism, we consider two algebras $\mathfrak{A}, \mathfrak{B} \in \mathfrak{B}(K, F)$ and the elements $a, b \in \mathrm{Z}$ corresponding to them. In other words: let $a E_{\phi}$ and $b E_{\phi}$ represent $\mathfrak{A}$ and $\mathfrak{B}$, respectively, in $K_{F}$. It follows, by Theorem 1.4 , that $\mathfrak{A} \otimes \mathfrak{B}$ is represented by $a E_{\phi} \otimes b E_{\phi}$ in $K_{F} \otimes K_{F}$, and the preceding lemma, in view of Theorem 1.3 , implies that $(a b) E_{\phi}$ represents an algebra $\mathfrak{E}$ similar to $\mathfrak{A} \otimes \mathfrak{B}$. In other words, this means that: $\mathfrak{A} \otimes \mathfrak{B} \rightarrow a b$, which proves the isomorphism.

\section{II}

5. Cohomology groups for arbitrary fields. Let $S, R$ be two commutative algebras over the field $C$. All tensor products used, henceforth, will be products relative to this field $C$ unless specified otherwise.

We set $R^{0}=C, R^{n}=R \otimes R \otimes \cdots \otimes R$ ( $n$-terms). Consider the isomorphism $\epsilon_{S, n}$ of the ring $R^{n} \otimes S$ into the ring $R^{n+1} \otimes S$ defined by

$$
\epsilon_{S, 0}(s)=1 \otimes s, \quad \epsilon_{S, n}(r \otimes s)=r \otimes 1 \otimes s, \quad r \in R^{n} \text { and } s \in S .
$$

In the notations of the preceding section, $\epsilon_{S, n}$ is the injection of $R^{n} \otimes S$ into $R^{n+1} \otimes S$.

We shall denote by $\left(R^{n} \otimes S\right)^{*}$ the multiplicative group of all regular elements of the ring $R^{n} \otimes S$, and we shall consider this group as a $J$-group, where the integers $J$ act on the group in the ordinary way of taking powers of elements. Note also that since $\epsilon_{S, n}$ is an isomorphism, it induces an isomorphism of the multiplicative group $\left(R^{n} \otimes S\right)^{*}$ into $\left(R^{n+1} \otimes S\right)^{*}$.

Let $\mathfrak{C}_{0}$ be the sequence of the groups $\left\{S^{*},(R \otimes S)^{*}, \cdots,\left(R^{n} \otimes S\right)^{*}, \cdots\right\}$. We shall turn the sequence $\mathfrak{C}_{0}$ into a $J$-cochain complex by introducing in $\mathfrak{C}_{0}$ a derivation mapping $\Delta_{S, n}:\left(R^{n} \otimes S\right)^{*} \rightarrow\left(R^{n+1} \otimes S\right)^{*}$ such that $\Delta_{S, n+1} \Delta_{S, n}=0$.

To this end we consider the sequence of sequences of groups $\mathfrak{C}_{i}$ $=\left\{\left[R^{n} \otimes\left(R^{i} \otimes S\right)\right]^{*}, n=0,1, \cdots\right\}$. To each of these sequences $\mathfrak{C}_{i}$ we associate the inclusion mappings $\epsilon_{R^{i}} \otimes S, n$, in which $S$ of definition (5.1) is replaced by $R^{i} \otimes S$.

Clearly for every $i \leqq n, \epsilon_{R^{i} \otimes S, n-i}$ maps $R^{n} \otimes S$ into $R^{n+1} \otimes S$ since $R^{n-i}$ $\otimes\left(R^{i} \otimes S\right)=R^{n} \otimes S$, but

$$
\epsilon_{R^{i} \otimes S, n-i}\left(r_{n} \otimes \cdots \otimes r_{i} \otimes \cdots r_{1} \otimes s\right)
$$

$$
=r_{n} \otimes \cdots \otimes r_{i+1} \otimes 1 \otimes r_{i} \otimes \cdots \otimes r_{1} \otimes s
$$


and we define the derivation $\Delta_{S, n}$ by induction as follows $\left({ }^{8}\right)$ :

$$
\Delta_{S, 0}=\epsilon_{S, 0} \text { and } \Delta_{S, n}=\epsilon_{S, n}-\Delta_{R \otimes S, n-1} .
$$

The induction is based on the fact that $\Delta_{S, n-1}$ is defined for all rings. A straight forward computation shows that:

(5.4) $\Delta_{S, n}=\epsilon_{S, n}-\epsilon_{R \otimes S, n-1}+\cdots+(-1)^{n} \epsilon_{R^{n} \otimes S, 0}=\sum_{i=0}^{n}(-1)^{i} \epsilon_{R^{i} \otimes S, n-i}$.

We now prove

THEOREM 5.1. The sequence

$$
S^{*} \stackrel{\Delta_{0}}{\rightarrow}(R \otimes S)^{*} \stackrel{\Delta_{1}}{\rightarrow} \cdots \rightarrow\left(R^{n} \otimes S\right)^{*} \stackrel{\Delta_{n}}{\rightarrow} \cdots
$$

where $\Delta_{n}=\Delta_{S, n}$ is a J-cochain complex, i.e., $\Delta_{n+1} \Delta_{n}=0$.

Proof. First we show that

$$
\epsilon_{S, n+1} \Delta_{S, n}+\Delta_{S, n+1} \epsilon_{S, n}=\epsilon_{S, n+1} \epsilon_{S, n} .
$$

Indeed, it follows by (5.2) that $\epsilon_{S, n+1} \epsilon_{R}^{i} \otimes S, n-i=\epsilon_{R}^{i+1} \otimes S,(n+1)-(i+1) \epsilon_{S, n}$ since both yield the isomorphism:

$$
r_{n} \otimes \cdots \otimes r_{i} \otimes \cdots \otimes r_{1} \otimes s
$$

$$
\rightarrow 1 \otimes r_{n} \otimes \cdots \otimes r_{i+1} \otimes 1 \otimes r_{i} \otimes \cdots \otimes r_{1} \otimes s .
$$

Hence, we obtain by (5.4) that

$$
\begin{aligned}
\epsilon_{S, n+1} \Delta_{S, n}+\Delta_{S, n+1} \epsilon_{S, n} & =\sum_{i=0}^{n}(-1)^{i} \epsilon_{S, n+1} \epsilon_{R^{i} \otimes S, n-i}+\sum_{i=0}^{n+1}(-1)^{i+1} \epsilon_{R^{i} \otimes S, n+1-i} \epsilon_{S, n} \\
& =\sum_{i=0}^{n} \epsilon_{R^{i+1} \otimes S, n-i} \epsilon_{S, n}+\sum_{i=0}^{n+1}(-1)^{i} \epsilon_{R^{i} \otimes S, n+1-i} \epsilon_{S, n} \\
& =\epsilon_{S, n+1} \epsilon_{S, n}, \text { q.e.d. }
\end{aligned}
$$

Next we show by induction that $\Delta_{S, n+1} \Delta_{S, n}=0$ for all $n \geqq 0$ and all rings $S$. Indeed for $n=0$ :

$$
\Delta_{S, 1} \Delta_{S, 0}=\left(\epsilon_{S, 1}-\epsilon_{R \otimes S, 0}\right) \epsilon_{S, 0}=0
$$

since both $\epsilon_{S, 1}$ and $\epsilon_{R \otimes S, 0}$ induce the same mappings on the elements $\epsilon_{S, 0}(s)$ $=1 \otimes s, s \in S$.

Suppose $\Delta_{S, n} \Delta_{S, n-1}=0$ for all rings $S$, then it follows by (5.4) and (a) that:

$$
\begin{aligned}
0 & =\Delta_{R \otimes S, n} \Delta_{R \otimes S, n-1}=\left(\epsilon_{S, n+1}-\Delta_{S, n+1}\right)\left(\epsilon_{S, n}-\Delta_{S, n}\right) \\
& =\epsilon_{S, n+1} \epsilon_{S, n}-\Delta_{S, n+1} \epsilon_{S, n}-\epsilon_{S, n+1} \Delta_{S, n}+\Delta_{S, n+1} \Delta_{S, n}=\Delta_{S, n+1} \Delta_{S, n}, \text { q.e.d. }
\end{aligned}
$$

(8) Since the groups of the complex $C_{0}$ are multiplicative groups, this definition means that: $\Delta_{S n}(a)=\epsilon S, n(a) \Delta_{R \otimes S, n-1}(a)^{-1}$. 
We shall denote the complex defined in (5.5) by $\mathcal{C}(S, R)$.

REMARK 5.1. Clearly the preceding theorem remains true if one replaces the ring $S$ by a multiplicative group $\mathcal{G}$ and $\left(R^{n} \otimes S\right)^{*}$ by the $R^{n *} \times \mathcal{G}$ where $\times$ denotes the direct product of groups.

We shall apply the preceding results to the case $S=K$ is a field extension of $C$ of degree $n$, and where $R=F$ is an arbitrary field extension of $C$. In this setting we obtain the complex $\mathfrak{C}(K, F)$ :

$$
K^{*} \rightarrow(F \otimes K)^{*} \rightarrow \cdots \rightarrow\left(F^{n} \otimes K\right)^{*} \rightarrow \cdots
$$

Taking $S=C$, we obtain the complex $\mathfrak{e}(C, F)$ which we simply denote by $\mathfrak{e}(F)$. In this complex we may set instead of the elements of $C=S$ the unit, since the tensor product is relative to $C$. We thus obtain the following complex:

$$
C^{*} \rightarrow F^{*} \rightarrow F^{2 *} \rightarrow \cdots \rightarrow F^{n *} \rightarrow \cdots
$$

Actually, the complex $\mathfrak{e}(F)$ is a subcomplex of $\mathfrak{e}(K, F)$. Indeed, the groups $\left(F^{n} \otimes K\right)^{*}$ of $\mathfrak{e}(K, F)$ contain the respective group $F^{n *}$ of $\mathfrak{e}(F)$; and the restriction of $\Delta_{K, n}$ to $F^{n *}$ is exactly $\Delta_{C, n}$. Consequently, these two complexes yield the following quotient complex $\mathfrak{e}(K, F) / \mathfrak{e}(F)$ :

$$
K^{*} / C^{*} \rightarrow(F \otimes K)^{*} / F^{*} \rightarrow \cdots \rightarrow\left(F^{n} \otimes K\right)^{*} / F^{n *} \rightarrow \cdots .
$$

We shall denote the derivation of this complex by $\bar{\Delta}_{n}$.

To these complexes, there corresponds the sequence of cohomological groups $H^{n}(\mathfrak{e}(K, F)), H^{n}(\mathfrak{e}(F))$ and $H^{n}(\mathfrak{e}(K, F) / C(F))$. The cohomology groups $H^{n}(\cdot)$ being defined as:

$$
H^{n}(\cdot)=\text { Kernel } \Delta_{n+1} / \text { Image } \Delta_{n}, \quad n=0,1, \cdots .
$$

where $\Delta_{n}=\Delta_{K, n}, \Delta_{C, n}, \bar{\Delta}_{n}$, respectively $\left(^{(9)}\right.$.

The results of the first part of this paper yield an interpretation of the first cohomology group of the quotient complex $\mathfrak{e}(K, F) / \mathfrak{e}(F)$. Namely,

THEOREM 5.2. If $K$ is a finite algebraic extension of the field $C$, then the Brauer group $\mathfrak{B}(K, F)$ of all central simple algebras split both by $K$ and by $F$ is isomorphic with $H^{1}(\mathfrak{e}(K, F) / \mathfrak{e}(F))$.

Proof. To determine the first cohomology group of this complex we begin with comparing the mappings $\epsilon$ defined in the beginning of this section with the isomorphisms $\alpha, \beta$ and $\phi$ of Remark 4.1, which were used in the preceding section.

Comparing (5.2) with Remark 4.1 we observe that since $\epsilon_{K, 1}(h \otimes k)$ $=h \otimes 1 \otimes k$ and $\epsilon_{F \otimes K, 0}(h \otimes k)=1 \otimes h \otimes k$ for $h \in F, k \in K$, we have the following:

( $\left.{ }^{9}\right)$ We use this definition for $H^{n}(\cdot)$ and not the classical definition of Kernel $\Delta_{n} /$ Image $\Delta_{n-1}$ for obvious reasons which will be seen later. 


$$
\epsilon_{F \otimes K, 0}=\phi \text { and } \epsilon_{K, 1} \text { is the injection of } F \otimes K \text { into } F^{2} \otimes K \text {. }
$$

Similarly, one observes that

(5.8b) $\epsilon_{F^{2} \otimes K, 0}=\alpha ; \epsilon_{F \otimes K, 1}=\beta$ and $\epsilon_{K, 2}$ is the injection of $F^{2} \otimes K$ into $F^{3} \otimes K$.

We turn now to the proof of the theorem. By definition $H^{1}(\mathfrak{e}(K, F) / \mathfrak{e}(F))$ $=$ Kernel $\Delta_{2} /$ Image $\Delta_{1}$. It follows by (5.4) that Kernel $\Delta_{2}$ contains all residue classes $\bar{a} \in\left(F^{2} \otimes K\right)^{*} / F^{2 *}$ such that

$$
\Delta_{2}(\bar{a})=\epsilon_{K, 2}(\bar{a}) \epsilon_{F \otimes K, 1}(\bar{a})^{-1} \epsilon_{F^{2} \otimes K, 0}(\bar{a})=\overline{1},
$$

where the unit $\overline{1}$ is the unit of the quotient group $\left(F^{3} \otimes K\right) /{ }^{*} F^{3 *}$. Let $a \in\left(F^{2} \otimes K\right)^{*}$ be an element representing the class $\bar{a}$, then the last condition on $\bar{a}$ is readily seen to be equivalent to the following condition on $a$ :

$$
\epsilon_{K, 2}(a) \epsilon_{F \otimes K, 1}(a)^{-1} \epsilon_{F^{2} \otimes K, 0}(a)=t \text { for some } t \in F^{*} .
$$

This condition is equivalent to (4.1). Indeed, replace in this condition the respective $\epsilon^{\prime}$ 's by $\alpha$ and $\beta$ in accordance with (5.8b), and one obtains $a\left(a^{\beta}\right)^{-1} a^{\alpha}$ $=t$ which is condition (4.1).

Thus, it follows that Kernel $\Delta_{2}=\left(\mathrm{Z}, F^{2 *}\right) / F^{2 *}$ where $\mathrm{Z}$ has the same meaning as in the preceding section: i.e., $Z$ contains all elements of $\left(F^{2} \otimes K\right)^{*}$ satisfying (4.1). Since $Z \supseteq F^{2 *}$, it follows that Kernel $\Delta_{2}=\mathcal{Z} / F^{2 *}$.

Now, Image $\Delta_{1}$ consists of all classes $\bar{a} \in\left(F^{2} \otimes K\right) / F^{2 *}$ which are of the form

$$
\bar{a}=\Delta_{1}(\bar{p})=\epsilon_{K, 1}\left(\bar{p} \epsilon_{F \otimes K, 0}(\bar{p})^{-1} \text { for some } \bar{p} \in(F \otimes K)^{*} / F^{*} .\right.
$$

If $a \in\left(F^{2} \otimes K\right)^{*}$ represents $\bar{a}$ and $p \in(F \otimes K)^{*}$ represents $\bar{p}$, then the last condition on $\bar{a}$ is equivalent to

$$
a=\epsilon_{K, 1}(p) \epsilon_{F \otimes K, 0}(p)^{-1} r \text { for some } r \in F^{*} .
$$

This is equivalent to the fact that $a$ satisfies (4.3) with $q=p^{-1}$. Indeed, by replacing $\epsilon_{F \otimes K, 0}$ by $\phi$, we obtain by (5.8a) that $a=p\left(p^{\phi}\right)^{-1} r$ which is condition (4.3). This clearly yields that Image $\Delta_{1}=\left(B, F^{2 *}\right) / F^{2 *}$ where $B$, as in the preceding section, is the group of all elements satisfying (4.3). Since $B \supseteq F^{2 *}$, it follows that: Image $\Delta_{1} \cong ß / F^{2 *}$. Consequently,

$$
H^{1}(\mathfrak{e}(K, F) / \mathfrak{e}(F)) \cong\left(\mathcal{Z} / F^{2 *}\right) /\left(\mathbb{B} / F^{2 *}\right) \cong Z / B \cong \mathfrak{B}(K, F),
$$

by Theorem 4.2 .

The symmetric definition of $\mathfrak{B}(K, F)$ readily yields, by the preceding theorem, that

Corollary 5.1. If $K$ and $F$ are finite algebraic extensions of $C$ then $H^{1}(\mathfrak{e}(K, F) / \mathfrak{e}(F)) \cong H^{1}(\mathfrak{e}(F, K) / \mathfrak{e}(K))$.

We intend now to represent the Brauer group $\mathfrak{B}(F)$ of all algebras split by $F$, by the cohomology groups of the complex $\mathfrak{e}(F)$, and first we obtain a 
result which is parallel to the triviality of certain known cohomology groups:

THEOREM 5.3. Let $K$ be an algebraic extension of an infinite field $C$, then $H^{1}(\mathfrak{e}(K, F))=1$.

Proof. By definition $H^{1}(\mathfrak{e}(K, F))=$ Kernel $\Delta_{2} /$ Image $\Delta_{1}$ and Kernel $\Delta_{2}$ contains the set of all $r \in\left(K \otimes F^{2}\right)^{*}$ which satisfy $\Delta_{2}(r)=\epsilon_{K, 2}(r) \epsilon_{F \otimes K, 1}(r)^{-1}$ $\epsilon_{F^{2} \otimes K, 0}(r)=1$, or equivalently, $\epsilon_{F \otimes K, 0}(r)=\epsilon_{K, 2}(r) \epsilon_{F^{2} \otimes K, 1}(r)$. Using (5.8b), this relation obtains the simple relation: $r r^{\alpha}=r^{\beta}$.

Let $\left\{h_{i}\right\}$ be a $C$-base of $F$. Since $r \in F^{2} \otimes K$, we have the following expression for $r: r=\sum h_{i} h_{j}^{\phi} k_{i j}$. Hence, one readily obtains, by Remark 4.1 that:

$$
r^{\alpha}=\sum h_{i}^{\phi} h_{j}^{\psi} k_{i j} \quad \text { and } \quad r^{\beta}=\sum h_{i} h_{j} k_{i j} .
$$

Put $u_{j}=\sum h_{i}^{\phi} k_{i j}$ and $v_{j}=\sum h_{i} k_{i j}$. Then the condition that $r r^{\alpha}=r^{\beta}$ means that $r\left(\sum u_{j} h_{j}\right)=\sum v_{j} h_{j}$. Now, $r, u_{j}$ and $v_{j}$ belong to $F^{2} \otimes K$ and $\left\{h_{j}^{\psi}\right\}$ is an $F^{2} \otimes K$-base of $F^{3} \otimes K$; hence $r u_{j}=v_{j}$ must hold for all $j$. Consequently, $r\left(\sum u_{j} x_{j}\right)=\sum v_{j} x_{j}$ for all $x_{j} \in C$.

Let $s=\sum v_{j} x_{j}=\sum h_{i} x_{j} k_{i j} \in F \otimes K$, then the last relation yields that $r s^{\phi}=s$ for some $s \in F \otimes K$. Coming back to the notation in the $\epsilon$ 's we obtain by (5.8a) that $r \epsilon_{K, 1}(s)=\epsilon_{F \otimes K, 0}(s)$, which will show that $r \in$ Image $\Delta_{1}$ if we can determine $x_{i}$ so that $s$ is a regular element, i.e. $s \in(F \otimes K)^{*}$. Consequently we shall obtain that $H^{1}(\mathfrak{C}(K, F))=1$ which we need. Indeed, $\sum v_{i} x_{i}$ clearly belongs to some finite algebraic extension $K^{\prime}$ of $C$. Now, Norm $\left(\sum v_{i} x_{i}\right)$ $=f\left(x_{i}\right)$ (where the Norm $=$ Norm $\left(K^{\prime} \otimes F / F\right)$ ), is a polynomial in the $\left\{x_{i}\right\}$ with coefficients in $F$. The polynomial $f\left(x_{i}\right) \not \equiv 0$, since in the ring $F^{2} \otimes K$ we know that Norm $r=\operatorname{Norm}\left(\sum v_{i} h_{i}^{\psi}\right)=f\left(h_{i}^{\psi}\right)$ is a regular element. Assuming that $C$ is an infinite field, we are able to find $x_{i}=c_{i} \in C$, so that $\sum v_{i} c_{i}$ is a regular element in $K \otimes F$, and the proof of the theorem is completed.

We are now in a position to show that the Brauer group $\mathfrak{B}(F)$ of all simple algebras split by $F$ satisfies:

THEOREM 5.4. If $F$ is a finite extension of $C$ then $\mathfrak{B}(F) \cong H^{2}(\mathfrak{e}(F))$.

Proof. We apply Theorem 5.2 for the case $K=F$. Consider the exact sequence:

$$
1 \rightarrow \mathfrak{e}(F) \stackrel{i}{\rightarrow} \mathfrak{e}(F, F) \stackrel{j}{\rightarrow} \mathfrak{e}(F, F) / \mathfrak{e}(F) \rightarrow 1 .
$$

By $[4$, p. 60$]$, this sequence leads to a cohomology sequence, of which we consider only the first terms:

$$
H^{1}(\mathfrak{e}(F, F)) \stackrel{j^{*}}{\rightarrow} H^{1}(\mathfrak{e}(F, F) / \mathfrak{e}(F)) \stackrel{\Delta^{*}}{\rightarrow} H^{2}(\mathfrak{e}(F)) \stackrel{i^{*}}{\rightarrow} H^{2}(\mathfrak{e}(F, F)) .
$$

The first group $H^{1}(\mathfrak{e}(F, F))=1$ by Theorem 5.3. Next, we prove that $i^{*}$ maps $H^{2}(\mathfrak{e}(F))$ onto the identity of $H^{2}(\mathfrak{e}(F, F))$. Indeed, 


$$
H^{2}(\mathfrak{e}(F))=\text { Kernel } \Delta_{3} / \text { Image } \Delta_{2} ;
$$

hence, if $r \in$ Kernel $\Delta_{2}, r \in F^{3 *}$, then it follows by definition of $\Delta_{3}=\Delta_{C, 3}$ that

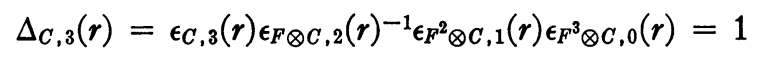

or equivalently

$$
\epsilon_{C, 3}(r)=\epsilon_{F \otimes C, 2}(r) \epsilon_{F^{2} \otimes C, 1}(r)^{-1} \epsilon_{F^{3} \otimes C, 0}(r) .
$$

Now it was pointed out in the beginning of this section that $\epsilon_{C, n}$ is the injection of $F^{n} \otimes C$ into $F^{n+1} \otimes C$. In our case $\epsilon_{C, 3}$ is exactly the injection $i^{*}$ we are dealing with. On the other hand, one readily verifies that by (5.2) that $\epsilon_{F^{n} \otimes C, l}=\epsilon_{F^{n-1} \otimes F, l}$ for all $l=0,1, \cdots, n-1$. It follows, therefore, that

$$
i^{*}(r)=\epsilon_{F, 2}(r) \epsilon_{F \otimes F, 1}(r)^{-1} \epsilon_{F^{2} \otimes F, 0}(r)=\Delta_{F, 2}(r) \epsilon \text { Image } \Delta_{F, 2} .
$$

Consequently, $i^{*}$ maps $H^{2}(\mathfrak{e}(F))$ onto the identity of $H^{2}(\mathfrak{e}(F, F))$.

Thus (5.9) is actually the following exact sequence:

$$
1 \rightarrow H^{1}(\mathcal{e}(F, F) / \mathfrak{e}(F)) \stackrel{\Delta^{*}}{\rightarrow} H^{2}(\mathfrak{e}(F)) \rightarrow 1 .
$$

The exactness of this sequence proves that $H^{2}(\mathfrak{e}(F)) \cong H^{1}(\mathfrak{e}(F, F) / \mathfrak{e}(F))$, and the latter is isomorphic with $\mathfrak{B}(F)$ by Theorem 5.2.

REMARK 5.2. Theorem 5.4 is valid also for infinite algebraic extensions of $C$. Since Theorem 5.3 and the preceding proof hold for infinite extensions, we still obtain that $H^{1}(\mathfrak{C}(F, F) / \mathfrak{e}(F)) \cong H^{2}(\mathfrak{C}(F))$. The weak point of the preceding proof is the application of Theorem 5.2, which was proved only for finite extensions $K$ of $C$. In order to fill this gap it remains to extend Theorem 5.2 to infinite extensions $K$ of $C$.

Indeed, if $\bar{a} \in H^{1}(\mathfrak{e}(K, F) / \mathfrak{e}(F))$ and $K$ is not necessarily finite over $C$, then one readily verifies that $\bar{a} \in H^{1}\left(\mathfrak{e}\left(K^{\prime}, F\right) / \mathcal{e}(F)\right)$ for some finite extension $K^{\prime}$ of $C, K^{\prime} \subseteq K$. Hence, by Theorem 5.2, it follows that $\bar{a}$ corresponds to a central simple algebra $\mathfrak{A} \in \mathfrak{B}\left(K^{\prime}, F\right) \subseteq \mathfrak{B}(K, F)$.

Conversely, if $\mathfrak{A}$ is a finite central simple algebra $\in \mathfrak{B}(K, F)$, then $\mathfrak{A}$ is split also by a field $K^{\prime} \subseteq K$ and $\left(K^{\prime}: C\right)<\infty$; i.e., $\mathfrak{A} \in \mathfrak{B}\left(K^{\prime}, F\right)$ for some finite extension $K^{\prime}$ of $C$. Hence, by Theorem 5.2, $\mathfrak{A}$ corresponds to some $\bar{a} \in H^{1}\left(\mathfrak{e}\left(K^{\prime}, F\right) / \mathfrak{e}(F)\right)$. Clearly, $\mathfrak{e}\left(K^{\prime}, F\right)$ can be considered as a subcomplex of $\mathfrak{e}(K, F)$ and, therefore, $\bar{a} \in H^{1}(\mathfrak{e}(K, F) / \mathfrak{e}(F))$. It is not difficult now, in view of Theorem 5.2, to prove that this correspondence between

$$
H^{1}(\mathfrak{e}(K, F) / \mathfrak{e}(F))
$$

and $\mathfrak{B}(K, F)$ is an isomorphism.

The proof of this remark shows in fact that if $K$ is a union of a linearly ordered set of field $K_{\alpha}$, then both $H^{1}(\mathfrak{e}(K, F) / \mathfrak{e}(F))$ and $\mathfrak{B}(K, F)$ are limit groups of $H^{1}\left(\mathfrak{e}\left(K_{\alpha}, F\right) / \mathfrak{e}(F)\right)$ and $\mathfrak{B}\left(K_{\alpha}, F\right)$, respectively. 
Theorem 5.4 is the unifying frame of both the classical results on the representation of the Brauer group of the algebras split by a normal extension and on the Brauer group of the algebras split by a purely inseparable extension of exponent 1. The proof of this fact is the object of the following sections.

We shall deal from now on only with the complex $\mathfrak{e}(F)$, and we shall denote its cohomology groups by $H^{n}(F)$ or by $H^{n}(F / C)$, and its derivation will be denoted by $\Delta_{n}$.

We shall treat separately the two cases: (1) $F$ be a normal extension of $C$, (2) $F$ be a purely inseparable extension of $C$ of exponent 1 .

6. Normal fields. In this section we assume that $F$ is a finite normal extension of $C$ with a group of automorphisms $G$. Let $\alpha_{1}, \cdots, \alpha_{m}$ be the automorphisms of $F$ over $C$. First we determine the structure of the ring $F^{n}$.

Lemma 6.1. $F^{2}=F e_{\alpha_{1}} \oplus \cdots \oplus F e_{\alpha_{m}}$ where $e_{\alpha}$ are primitive idempotents in $F^{2}$ satisfying: $(1 \otimes h) e_{\alpha}=\alpha(h) e_{\alpha}$.

This result is well known (e.g. [10] and [5]), but we shall produce here a different proof which will yield some additional information on the idempotents $e_{\alpha}$.

Proof. We consider $F^{2}$ both as a ring and as a left $F$-module by setting $h r=(h \otimes 1) r$, for $h \in F$ and $r \in F^{2}$.

Let $N$ be the ideal in $F^{2}$ generated by the set $\{h \otimes 1-1 \otimes h ; h \in F\}$. Since $F$ is a separable extension of $C$, the ring $F^{2}$ is semi-simple. Hence $F^{2}=N \oplus N_{1}$, where $N_{1}$ is an ideal in $F^{2}$. It follows now from the definition of $N$ that $N_{1} \cong F^{2} / N \cong F$; hence, $N_{1}$ is a minimal ideal in $F^{2}$ which implies that $N_{1}=F e$ where $e$ is a primitive idempotent. Clearly, the definition of $N$ yields that:

$$
h e=(h \otimes 1) e=(1 \otimes h) e .
$$

For a fixed $\alpha \in G$, the mapping $\alpha \otimes 1$ of $F \otimes F$ is readily seen to be an isomorphism of the latter over $1 \otimes F$. Hence, the element $e_{\alpha}=(\alpha \otimes 1) e$ is also a primitive idempotent of $F^{2}$. Furthermore, since $(h \otimes 1) e=(1 \otimes h) e$ it follows that

$$
\alpha(h) e_{\alpha}=(\alpha \otimes 1)[(h \otimes 1) e]=(\alpha \otimes 1)[(1 \otimes h) e]=(1 \otimes h) e_{\alpha} .
$$

In particular this relation implies that all idempotents $e_{\alpha}$ are different. The number of these idempotents is equal to the order of $\mathcal{G}=(F: C)=\left(F^{2}: F\right)$. Hence, clearly, $F^{2}=F e_{\alpha_{1}} \oplus \cdots \oplus F e_{\alpha_{m}}$, q.e.d.

We shall denote in the following theorem: $h^{(i)}=1 \otimes \cdots \otimes h \otimes \cdots \otimes 1$, where $h \in F$ stands in $i$ th place and 1 elsewhere. $(\alpha)=\left(\alpha_{1}, \cdots, \alpha_{n}\right)$ will denote an $n$-tuple of elements $\alpha_{i} \in G$.

We now extend the result of the previous lemma and we show:

Lemma 6.2. $F^{n+1}=\sum F e_{(\alpha)}$, where the sum is direct and it ranges over all $n$-tuples $(\alpha)$ of elements of $\mathrm{G}$. The element $e_{(\alpha)}$ is a primitive idempotent which is uniquely determined by the condition: 


$$
h^{(i)} e_{\left(\alpha_{1}, \cdots, \alpha_{n}\right)}=\alpha_{1} \alpha_{2} \cdots \alpha_{i-1}(h) e_{\left(\alpha_{1}, \cdots, \alpha_{n}\right)},
$$$$
i=2, \cdots, n+1
$$

and by definition, $h^{(1)} e_{(\alpha)}=h e_{(\alpha)}$.

Proof. The proof is carried out by induction on $n$. The case $n=1$ has been proved in the preceding lemma. Suppose the lemma is true for $n$, then since $F^{n+1}=\sum F e_{(\alpha)},(\alpha)=\left(\alpha_{1}, \cdots, \alpha_{n}\right)$, it follows that $F^{n+2}=\sum F e_{(\alpha)} \otimes F$, and we shall show that

$$
F e_{(\alpha)} \otimes F=\sum_{\beta \in G} F e_{\left(\alpha_{1}, \cdots, \alpha_{n}, \gamma\right)}, \quad \text { where } \quad \gamma=\alpha_{n}^{-1} \alpha_{n-1}^{-1} \cdots \alpha_{1}^{-1} \beta .
$$

Indeed, let $\rho: F \otimes F \rightarrow F e_{(\alpha)} \otimes F$ be the isomorphism defined by: $\rho(h \otimes k)$ $=h e_{(\alpha)} \otimes k$. Note that $\rho$ is also an $F$-isomorphism between the two $F$-modules: $F \otimes F$ and $F e_{(\alpha)} \otimes F$, namely: $\rho(h x)=h \rho(x)$ for $h \in F$ and $x \in F^{2}$. It follows by the preceding lemma that $F \otimes F=\sum F e_{\beta}$, hence $F e_{(\alpha)} \otimes F=\sum F \rho\left(e_{\beta}\right)$, and clearly $\rho\left(e_{\beta}\right)$ is a primitive idempotent in $F^{n+2}$. Our next aim is to show that:

$$
\rho\left(e_{\beta}\right)=e_{\left(\alpha_{1}, \cdots, \alpha_{n}, \gamma\right)}, \text { where } \gamma=\alpha_{n}^{-1} \cdots \alpha_{1}^{-1} \beta .
$$

Now $e_{(\alpha)} \otimes 1$ is the unit of $F e_{(\alpha)} \otimes F$ and $\rho$ is an $F$-isomorphism, hence it follows by (6.2) that: for $i=1,2, \cdots, n+1$

$$
\begin{aligned}
h^{(i)} \rho\left(e_{\beta}\right) & =h^{(i)}\left(e_{(\alpha)} \otimes 1\right) \rho\left(e_{\beta}\right)=\left(h^{(i)} e_{(\alpha)} \otimes 1\right) \rho\left(e_{\beta}\right) \\
& =\left(\alpha_{1} \cdots \alpha_{i-1}(h) e_{(\alpha)} \otimes 1\right) \rho\left(e_{\beta}\right)=\alpha_{1} \alpha_{2} \cdots \alpha_{i-1}(h)\left(e_{(\alpha)} \otimes 1\right) \rho\left(e_{\beta}\right) \\
& =\alpha_{1} \alpha_{2} \cdots \alpha_{i-1}(h) \rho\left(e_{\beta}\right),
\end{aligned}
$$

and for $i=n+2$

$$
\begin{aligned}
h^{(n+2)} \rho\left(e_{\beta}\right) & =\rho\left[(1 \otimes h) e_{\beta}\right]=\rho\left[\beta(h) e_{\beta}\right]=\beta(h) \rho\left(e_{\beta}\right) \\
& =\alpha_{1} \alpha_{2} \cdots \alpha_{n} \gamma(h) \rho\left(e_{\beta}\right) \text { where } \gamma=\alpha_{n}^{-1} \alpha_{n-1}^{-1} \cdots \alpha_{1}^{-1} \beta,
\end{aligned}
$$

which proves (6.3). The proof of the rest of the lemma is now evident. We only point out that (6.2) implies that all the $e_{(\alpha)}$ are different and that their number is equal to $\left(F^{n+1}: F\right)$.

The main object of the present section is the following theorem:

Theorem 6.1. Let $F$ be a finite normal extension of $C$ with the Galois group of automorphism $G$, then $H^{n}(F)=H^{n}\left(F^{*}, G\right)$.

Proof. Let $C_{n}=\operatorname{Hom}_{J}\left(F^{*}, G^{n}\right)$ be the multiplicative group of all functions of $G^{n}$ into $F^{*}$. In the present proof we shall use the following definition for $H^{n}\left(F^{*}, G\right)$ :

The sequence

$$
C_{0} \stackrel{\delta^{0}}{\rightarrow} C_{1} \stackrel{\delta^{1}}{\rightarrow} \cdots \rightarrow C_{n} \stackrel{\delta^{n}}{\rightarrow} \cdots
$$

with the coderivation 


$$
\left(\delta^{n} f\right)\left(\alpha_{1}, \cdots, \alpha_{n+1}\right)=\alpha_{1}\left(f\left(\alpha_{2}, \cdots, \alpha_{n+1}\right)\right)
$$

$$
\cdot \prod_{i=1}^{n} f\left(\alpha_{1}, \cdots, \alpha_{i} \alpha_{i+1}, \cdots, \alpha_{n+1}\right)^{(-1)^{i}} f\left(\alpha_{1}, \cdots, \alpha_{n}\right)^{(-1)^{n-1}}
$$

yield the cohomology groups $H^{n}\left(F^{*}, G\right)=$ kernel $\delta^{n} /$ Image $\delta^{n-1}$.

We shall exhibit an isomorphism $\sigma$ between this complex and the complex $C(F)$ defined in (5.6b) but in which we omit the first member, namely:

$$
F^{*} \stackrel{\Delta_{1}}{\rightarrow} F^{2 *} \stackrel{\Delta_{2}}{\rightarrow} \cdots \rightarrow F^{n *} \stackrel{\Delta_{n}}{\rightarrow} \cdots
$$

In other words we shall define an isomorphism $\sigma: F^{n+1 *} \rightarrow \operatorname{Hom}_{J}\left(F^{*}, G^{n}\right)$ such that $\sigma \Delta_{n+1}=\delta^{n} \sigma$ for all $n \geqq 0$.

Since $F^{n+1}=\sum F e_{(\alpha)}$, it follows that for $a \in F^{n+1}$,

$$
a e_{\left(\alpha_{1}, \cdots, \alpha_{n}\right)}=f_{a}\left(\alpha_{1}, \cdots, \alpha_{n}\right) e_{\left(\alpha_{1}, \cdots, \alpha_{n}\right)} \text {, for some } f_{a}((\alpha)) \in F,
$$

and conversely, for any set of elements $f((\alpha)) \in F$ we define an element $a_{f} \in F^{n+1}$ by

$$
a_{f}=\sum_{(\alpha)} f((\alpha)) e_{(\alpha)}
$$

Clearly, $a \in F^{n+1 *}$, i.e., $a$ is a regular element, if and only if $f_{a}((\alpha)) \neq 0$ for all $(\alpha)$. Furthermore, since the idempotents $e_{(\alpha)}$ are orthogonal, the mappings $a \rightarrow f_{a}$ and $f \rightarrow a_{f}$ are inverse mappings between $F^{n+1}$ and the set of all functions of $g^{n}$ into $F$. The isomorphism $\sigma$ which we need is defined by:

$$
\sigma(a)=f_{a} \text { and, therefore, } \sigma^{-1}(f)=a_{f} .
$$

Thus, $\sigma$ is a one to one map of $F^{n+1 *}$ onto $\operatorname{Hom}_{J}\left(F^{*}, \mathrm{~g}^{n}\right)$. To prove that $\sigma$ is actually an isomorphism between these groups, we observe that:

$$
f_{a b}((\alpha)) e_{(\alpha)}=(a b) e_{(\alpha)}=a f_{b}((\alpha)) e_{(\alpha)}=f_{b}((\alpha)) f_{a}((\alpha)) e_{(\alpha)},
$$

which shows that $\sigma(a b)=\sigma(a) \sigma(b)$.

We turn now to the last stage in our proof which is to show that $\sigma \Delta_{n+1}$ $=\delta^{n} \sigma$. That is, for every $a \in F^{n+1}$ we must have

$$
\left(\delta^{n} f_{a}\right)\left(\alpha_{1}, \cdots, \alpha_{n+1}\right)=f_{\Delta_{n+1} a}\left(\alpha_{1}, \cdots, \alpha_{n+1}\right) .
$$

We begin the proof by computing the right side of this equation. It follows by (5.4) and (6.6) that:

$$
f_{\Delta_{n+1} a}((\alpha)) e_{(\alpha)}=\left(\Delta_{n+1} a\right) e_{(\alpha)}=\prod_{i=0}^{n+1} \epsilon_{i}(a)^{(-1)^{n+1-i}} e_{(\alpha)}
$$

$$
=\prod_{i=0}^{n+1} f_{\epsilon_{i}(a)}((\alpha))^{(-1)^{n+1-i}} e_{(\alpha)}
$$

where $\epsilon_{i}=\epsilon_{F^{n+1-i}},{ }_{i}$. 
First we compute the functions $f_{a, i}$ for elements of the form $a=h_{1} \otimes \ldots$ $\otimes h_{n+1}=h_{1}^{(1)} \cdots h_{n+1}^{(n+1)}$. We recall that $h_{i}^{(1)}=1 \otimes \cdots \otimes h_{i} \otimes \cdots \otimes 1$ with $h_{i}$ standing in the $i$ th place.

Now $\quad \epsilon_{i}(a)=\epsilon_{F}^{n+1-i}, i(a)=h_{1} \otimes \cdots \otimes h_{i} \otimes 1 \otimes h_{i+1} \otimes \cdots \otimes h_{n+1}=h_{1}^{(1)} \ldots$ $h_{i}^{(1)} h_{i+1}^{(1+2)} \cdots h_{n+1}^{(n+2)}$. It follows, therefore, by (6.2) that for $i=1,2, \cdots, n+1$

$$
\begin{aligned}
f_{\epsilon_{i}(a)}((\alpha)) e_{(\alpha)}=\epsilon_{i}(a) e_{(\alpha)}=h_{1}^{(1)} \cdots h_{i}^{(i)} h_{i+1}^{(i+2)} \cdots h_{n+1}^{(n+2)} e_{(\alpha)} \\
=h_{1}\left[\alpha_{1}\left(h_{2}\right)\right]\left[\alpha_{1} \alpha_{2}\left(h_{3}\right)\right] \cdots\left[\alpha_{1} \cdots \alpha_{i-1}\left(h_{i}\right)\right]\left[\alpha_{1} \cdots \alpha_{i-1} \alpha_{i} \alpha_{i+1}\left(h_{i+1}\right)\right] \\
\quad \cdot\left[\alpha_{1} \alpha_{2} \cdots \alpha_{n+1}\left(h_{n+1}\right)\right] e_{(\alpha)} \\
=f_{a}\left(\alpha_{1}, \alpha_{2}, \cdots, \alpha_{i} \alpha_{i+1}, \alpha_{i+2}, \cdots, \alpha_{n+1}\right) e_{\left(\alpha_{1}, \cdots, \alpha_{n+1}\right)},
\end{aligned}
$$

since, by (6.2),

$$
\begin{gathered}
a e_{\left(\alpha_{1}, \cdots, \alpha_{i} \alpha_{i}+1, \cdots \alpha_{n+1}\right)}=h_{1}^{(1)} h_{2}^{(2)} \cdots h_{n+1}^{(n+1)} e_{\left(\alpha_{1}, \cdots, \alpha_{i} \alpha_{i+1}, \cdots, \alpha_{n+1}\right)} \\
=h_{1}\left[\alpha_{1}\left(h_{2}\right)\right] \cdots\left[\alpha_{1} \cdots \alpha_{i-1}\left(h_{i}\right)\right]\left[\alpha_{1} \cdots \alpha_{i-1} \alpha_{i} \alpha_{i+1}\left(h_{i+1}\right)\right] \cdots \\
\cdot\left[\alpha_{1} \cdots \alpha_{n+1}\left(h_{n+1}\right)\right] .
\end{gathered}
$$

Since $f_{b}((\alpha))+f_{c}((\alpha))=f_{b+c}((\alpha))$, it follows readily that (6.10) is valid for all $a \in F^{n+1}$.

For $i=0$ and $a$ the element dealt with above, we obtain:

$$
\begin{aligned}
f_{\left(e_{0} a\right)}((\alpha)) e_{(\alpha)} & =\epsilon_{0}(a) e_{(\alpha)}=h_{1}^{(2)} h_{2}^{(3)} \cdots h_{n+1}^{(n+2)} e_{\left(\alpha_{1}, \cdots, \alpha_{n+1}\right)} \\
& =\left[\alpha_{1}\left(h_{1}\right)\right]\left[\alpha_{1} \alpha_{2}\left(h_{2}\right)\right] \cdots\left[\alpha_{1} \alpha_{2} \cdots \alpha_{n}\left(h_{n+1}\right)\right] e_{(\alpha)} \\
& =\alpha_{1}\left[\left(f_{a}\left(\alpha_{2}, \cdots, \alpha_{n+1}\right)\right] e_{(\alpha)},\right.
\end{aligned}
$$

since by (6.2) one obtains in a similar way that $f_{a}\left(\alpha_{2}, \cdots, \alpha_{n+1}\right)=h_{1}\left[\alpha_{2}\left(h_{2}\right)\right]$ $\cdots\left[\alpha_{2} \cdots \alpha_{n+1}\left(h_{n+1}\right)\right]$. For $i=n+1$,

$$
\begin{aligned}
f_{c_{n+1}(a)}((\alpha)) e_{(\alpha)} & =\epsilon_{n+1}(a) e_{(\alpha)}=h_{1}^{(1)} \cdots h_{n+1}^{(n+1)} e_{(\alpha)} \\
& =h_{1}\left[\alpha_{1}\left(h_{2}\right)\right] \cdots\left[\alpha_{1} \cdots \alpha_{n}\left(h_{n}\right)\right] e_{(\alpha)}=f_{a}\left(\alpha_{1}, \cdots, \alpha_{n}\right) e_{(\alpha)},
\end{aligned}
$$

and from the reason stated above it follows that (6.10a) and (6.10b) for all $a \in F^{n+1}$.

The required relation that $\sigma \Delta_{n+1}=\delta^{n} \sigma$ follows now, by inserting the three results (6.10), (6.10a) and (6.10b) in (6.9) and by comparing the outcome with (6.5). Consequently, $\sigma$ is a cochain isomorphism between the complexes (6.4) and (6.6) and it induces, therefore, the isomorphism $H^{n}\left(F^{*}, G\right) \cong H^{n}(F)$.

In particular, the last theorem together with Theorem 5.1 proves the classical representation of the Brauer group $\mathfrak{B}(F)$ of the central simple algebras split by $F$ :

Theorem 6.2. $\mathfrak{B}(F)=H^{2}\left(F^{*}, G\right)$. 
7. The inseparable case of exponent 1 . For the purpose of the present section we assume that $C$ is a field of characteristic $p \neq 0$ and that $F$ is a purely inseparable extension of $C$ of exponent 1.

Let $N_{n}$ be the ideal of the ring $F^{n}$ generated by the set $\left\{h-h^{(i)} ; h \in F\right\}$, and as in the preceding section $h^{(i)}=1 \otimes \cdots \otimes h \otimes \cdots \otimes 1$, where $h$ stands in the $i$ th place. As in the normal case one verifies that $F^{n} / N_{n} \cong F$, but in the present case $N_{n}$ is a nilpotent ideal, and for every $n \in N_{n}, n^{p}=0$. Indeed, let $n=\sum r_{i j}\left(h_{j}-h_{j}^{(i)}\right)$, then $n^{p}=\sum r_{i j}^{p}\left(h_{j}^{p}-\left(h_{j}^{p}\right)^{(i)}\right)=0$; since $F$ is a separable extension of $C$ of exponent 1, which implies that $h_{j}^{p}=c_{j} \in C$ and by the definition of the tensor product with respect to $C$, it follows that $c_{j}=c_{j}^{(i)}=0$.

Thus $N_{n}$ is the radical of the ring $F_{n}^{0}$. Let $N_{n}=\left\{1+n ; n \in N_{n}\right\}$, then $N_{n}^{0}$ is a multiplicative subgroup of $F^{n *}$. Moreover, since $F^{n}=F^{+} \oplus N_{n}^{+}$is a sum of the additive groups of the elements of $F$ and of the elements of $N_{n}^{+}$, it follows that every $r \in F^{n *}$ has the form $r=\alpha+n, \alpha \neq 0, \alpha \in F$ and $n \in N_{n}$. Hence, $r=\alpha\left(1+\alpha^{-1} n\right)=\alpha^{-1}(1+m)$ and $m \in N_{n}$. This proves that $F_{n}^{n *}=F^{*} N_{n}^{0}$; and it follows, therefore, that $F^{n *} / N_{n}^{0} \cong F^{*}$.

Returning back to the isomorphisms $\epsilon_{i}=\epsilon_{F^{n-1}, i}$ we note that these isomorphisms map also $N_{n}^{0}$ into $N_{n+1}^{0}$. Consequently, the coderivation $\Delta_{n}$ maps also $N_{n}^{0}$ into $N_{n+1}^{0}$ which means that the following sequence:

$$
1 \rightarrow N_{1}^{0} \stackrel{\Delta_{1}}{\rightarrow} N_{2}^{0} \stackrel{\Delta_{2}}{\rightarrow} \cdots \rightarrow N_{n}^{0} \stackrel{\Delta_{n}}{\rightarrow} \cdots
$$

forms a subcomplex of the complex $\mathfrak{C}(F)$ defined in (5.2). We shall denote this comples by $\mathrm{e}\left(N^{0}\right)$.

We thus have obtained an exact sequence:

$$
1 \rightarrow \mathfrak{e}\left(N^{0}\right) \rightarrow \mathfrak{e}(F) \rightarrow \mathfrak{e}(F) / \mathfrak{e}\left(N^{0}\right) \rightarrow 1 .
$$

It will be shown later that $H^{n}\left(\mathfrak{C}(F) / \mathfrak{C}\left(N^{0}\right)\right)=1$; hence, the cohomology sequence obtained by the preceding exact sequence:

$$
1=H^{n-1}\left(\mathfrak{e}(F) / \mathfrak{e}\left(N^{0}\right)\right) \rightarrow H^{n}\left(\mathfrak{e}\left(N^{0}\right)\right) \rightarrow H^{n}(\mathfrak{e}(F)) \rightarrow H^{n}\left(\mathfrak{e}(F) / C\left(N^{0}\right)\right)=1
$$

yield that:

THEOREM 7.1. $H^{n}\left(\mathfrak{C}\left(N^{0}\right)\right) \cong H^{n}(\mathfrak{C}(F))$.

To complete the proof of this theorem it remains to show that

$$
H^{n}\left(\mathfrak{e}(F) / \mathfrak{e}\left(N^{0}\right)\right)=1 .
$$

Indeed, the quotient complex $\mathcal{C}(F) / \mathcal{C}\left(N^{0}\right)$ contains the groups $F^{n *} / N_{n}^{0}$ for $n \geqq 1$, and 1 for $n=0$. These groups can be considered in a natural way as the multiplicative groups of the regular elements of $F^{n} / N_{n}$. The latter are isomorphic with $F$ by mapping: $h \rightarrow \bar{h}, h \in F$ and, where $\bar{h}$ denote the residue class mod $N_{n}$ represented by the element $h$. Now the mappings $\epsilon_{i}$ clearly induce an isomorphism between $F^{n} / N_{n}$ and $F^{n+1} / N_{n+1}$ which both are rings isomorphic with $F$. Furthermore, clearly the isomorphism, which is 
thus induced in $F$, leaves the elements of the field $C$ invariant; hence, since $F$ is purely inseparable extension of $C$, it follows that this isomorphism is the identity. Consequently, we obtain that $\epsilon_{i}(r) \equiv r\left(\bmod N_{n+1}\right)$ for every $r \in F^{n}$. In particular, one readily verifies that this implies that $\epsilon_{i}(r) \equiv r\left(\bmod N_{n+1}^{0}\right)$ for $r \in F^{n *}$.

It follows, therefore, by $(5.4)$ that $\epsilon_{i}(r) \equiv r\left(\bmod N_{n+1}^{0}\right)$ if $n$ is even and $\epsilon_{i}(r) \equiv 1\left(\bmod N_{n+1}^{0}\right)$ if $n$ is odd. In other words, the quotient complex

$$
C(F) / C\left(N^{0}\right)
$$

is isomorphic with a complex:

$$
1 \rightarrow 1 \rightarrow F^{*} \rightarrow F^{*} \ldots \rightarrow F^{*} \rightarrow \cdots
$$

in which the coderivation is alternatively the zero map: $r \rightarrow 1$, and the identity map: $r \rightarrow r$. Evidently, the cohomology groups of such a complex are trivial, which was required to show.

We can go further in simplifying the complexes in the present case by replacing the multiplicative groups by additive groups:

Let $N_{n}^{+}$denote the additive group of the elements of $N_{n}$ and we set $N_{0}^{+}=N_{1}^{+}=0$. Consider the mapping:

$$
\eta: n \rightarrow 1+n+\frac{n^{2}}{2 !}+\cdots+\frac{n^{p-1}}{(p-1) !} \quad(=\exp (n)), n \in N_{n} .
$$

Since the elements of $N_{n}$ are nilpotent elements of index $p$, i.e., $n^{p}=0$, one readily verifies that $\eta$ is an isomorphic mapping of the additive group $N_{n}^{+}$ onto the multiplicative group $N_{n}^{0}$, and its inverse is the mapping:

$$
\eta^{-1}: 1+m \rightarrow m-\frac{m^{2}}{2}+\cdots+(-1)^{p} \frac{m^{p-1}}{p-1}, \quad 1+m \in N_{n}^{0} .
$$

Since the $\epsilon_{i}$ are isomorphisms of $F^{n}$ into $F^{n+1}$. it follows readily, by the definition of $\eta$, that

$$
\epsilon_{i} \eta=\eta \epsilon_{i} .
$$

We now use the definition of $\Delta_{n}$ of (5.4) also for the additive groups $N_{n}^{+}$. Namely, we set:

$$
\Delta_{n}^{+}(r)=\epsilon_{n}(r)-\epsilon_{1}(r)+\cdots+(-1)^{n} \epsilon_{0}(r), \quad r \in N_{n}^{+},
$$

where $\epsilon_{i}=\epsilon_{F^{n-i}, i}$. We thus obtain a complex:

$$
0 \rightarrow N_{1}^{+} \stackrel{\Delta_{1}^{+}}{\longrightarrow} \cdots \rightarrow N_{n} \stackrel{\Delta_{n}^{+}}{\longrightarrow} \cdots,
$$

which we shall denote by $\mathfrak{e}\left(N^{+}\right)$. Next we show: 
Lemma 7.1. The isomorphism $\eta$ is a complex isomorphism of $\mathfrak{e}\left(N_{n}^{+}\right)$onto $\mathfrak{e}\left(N^{0}\right)$, and hence, $H^{n}\left(\mathfrak{e}\left(N^{+}\right) \cong H^{n}\left(\mathfrak{e}\left(N^{0}\right)\right)\right.$.

Proof. It was pointed out already that $\eta$ is an isomorphism of $N_{n}^{+}$onto $N_{n}^{0}$. Hence, to complete the proof of this lemma one has to show that $\eta \Delta_{n}^{+}$ $=\Delta_{n} \eta$. Indeed, first we note that $\eta(x+y)=\eta(x) \eta(y)$, hence it follows by (7.2) that for $r \in N_{n}^{+}$,

$$
\begin{aligned}
\left(\eta \Delta_{n}^{+}\right)(r) & =\eta\left(\sum_{i=0}^{n}(-1)^{n-i} \epsilon_{i}(r)\right)=\prod_{i=1}^{n}\left(\eta \epsilon_{i}(r)\right)^{(-1)^{n-i}} \\
& =\prod_{i=1}^{n}\left(\epsilon_{i} \eta(r)\right)^{(-1)^{n-i}}=\left(\Delta_{n} \eta\right)(r) \text {. q.e.d. }
\end{aligned}
$$

We note also that the definition $\Delta_{n}^{+}$in (7.3) is good also for all $r \in F^{n+}$, where $F^{n+}$ denotes the additive group of the elements of $F^{n}$. Hence, the mapping $\Delta_{n}^{+}$may be considered as a coderivation of the complex:

$$
0 \rightarrow F^{+} \stackrel{\Delta_{1}}{\rightarrow} F^{2+} \rightarrow \cdots \rightarrow F^{n+} \stackrel{\Delta_{n}}{\rightarrow} \cdots .
$$

We shall denote this complex by $\mathfrak{e}\left(F^{+}\right)$, and its cohomology groups will be denoted by $H^{n}\left(F^{+}\right)$. The passage from the cohomology groups $H^{n}\left(\mathfrak{e}\left(N^{+}\right)\right)$to the new cohomology groups $H^{n}\left(F^{+}\right)$is similar, but in the opposite direction, to the passage from $H^{n}\left(F^{*}\right)$ to $H^{n}\left(\mathfrak{e}\left(N^{0}\right)\right)$. Namely, the complex $\mathfrak{e}\left(N^{+}\right)$is readily seen to be a subcomplex of $\mathfrak{e}\left(F^{+}\right)$. The quotient complex $\mathfrak{C}\left(F^{+}\right) / \mathfrak{e}\left(N^{+}\right)$ contains the groups $F^{n+} / N_{n}^{+} \cong F^{+}$, and as in the multiplicative case, this complex is isomorphic with a complex: $0 \rightarrow F^{+} \rightarrow F^{+} \rightarrow \cdots$ in which the coderivation is alternatively the zero map and the identity map, and, consequently its cohomology groups are all trivial. Hence, as before, the exact sequence:

$$
0 \rightarrow \mathfrak{e}\left(N^{+}\right) \rightarrow \mathfrak{e}\left(F^{+}\right) \rightarrow \mathfrak{e}\left(F^{+}\right) / \mathfrak{e}\left(N^{+}\right) \rightarrow 0
$$

yield that

$$
0=H^{n-1}\left(\mathfrak{e}\left(F^{+}\right) / \mathfrak{e}\left(N^{+}\right)\right) \rightarrow H^{n}\left(\mathfrak{e}\left(N^{+}\right)\right) \rightarrow H^{n}\left(F^{+}\right) \rightarrow H^{n}\left(\mathfrak{e}\left(F^{+}\right) / \mathfrak{e}\left(N^{+}\right)\right)=0
$$

whence:

THEOREM 7.2. $H^{n}\left(F^{+}\right) \cong H^{n}\left(\mathfrak{e}\left(N^{+}\right)\right) \cong H^{n}(F)$.

We intend now to show that $H^{n}\left(F^{+}\right)$is isomorphic with a subgroup of the restricted cohomology group $H_{*}^{n}(F, \mathfrak{L})$ where $\mathscr{L}$ is the Lie-algebra of derivation of $F$ over $C$, and we begin with introducing some basic notions.

A derivation $D$ of $F$ over $C$ is a $C$-endomorphism of the additive group $F^{+}$satisfying: $D(x y)=D(x) y+x D(y)$. The set $\&$ of all derivations of $F$ over $C$ form a restricted Lie-algebra in the sense of Jacobson [9]. That is: $\mathscr{L}$ is a $C$-space closed with respect to the Lie-bracket: $\left[D_{1}, D_{2}\right]=D_{1} D_{2}-D_{2} D_{1}$, and 
with respect with the $p$-mapping: $D \rightarrow D^{p}$.

The $u$-algebra $\mathfrak{U}_{L}$ of $L$ is defined as follows (see e.g. [6]): Let $T_{L}$ be the tensor algebra of $L$; namely, $T_{L}=\sum_{i+0}^{\infty} T_{L^{i}}$ where $T_{L^{0}}=C$ and $T_{L^{i}}$ is the $i$-fold tensor product $L \otimes L \otimes \cdots \otimes L$, and the multiplication in $T_{L}$ is defined by the canonical map: $T_{L} i \otimes T_{L} \rightarrow T_{L}{ }^{i+i}$. Let $P_{L}$ be the ideal in $T_{L}$ generated by the elements $\{x \otimes y-y \otimes x-[x, y] ; x, y \in L\}$ and by the elements $\left\{x^{p}-x^{[p]} ; x \in L\right\}$, where $x^{p}$ denotes the $p$ th power of the element $x \in T_{L}$, and $x^{[p]} \in L$ is the derivation of $F$ obtained as the $p$ th power of $x$ when the latter is considered as a linear operator of $F$. Then, the $u$-algebra $\mathfrak{u}_{L}=T_{L} / P_{L}$.

We set, as in [6], $\mathfrak{U}_{L}^{+}=T_{L}^{+} / P_{L}$, where $T_{L}^{+}=\sum_{i=1}^{\infty} T_{L}{ }^{i}$. The elements of the field $C$ are identified with $\left(C, P_{L}\right) / P_{L}$. We shall denote by $\epsilon$ the canonical map of $\mathfrak{U}_{L} \rightarrow \mathfrak{U}_{L} / \mathfrak{U}_{L}^{+}$, and for every $u \in \mathfrak{U}_{L}$ we have: $\epsilon(u) \in C$ and $u-\epsilon(u) \in \mathfrak{U}_{L}^{+}$.

The additive group $F^{+}$can be considered in a natural way as a restricted $\mathfrak{L}$-module, or equivalently (by [6]) as a $\mathfrak{U}_{L}$-module. This is achieved by setting:

$$
\begin{gathered}
c(h)=c h \text { for } h \in F \text { and } c \in T_{L^{0}}=C, \text { and } \\
{\left[u_{1} \otimes \cdots \otimes u_{i}\right](h)=u_{1} u_{2} \cdots u_{i}(h), u_{i} \in \mathcal{L},}
\end{gathered}
$$

and by extending it linearly for all elements of $T_{L}$. Since $P_{L}(F)=0$, it follows that the preceding definition turns $F$ into a $\mathfrak{U}_{L}$-module.

In particular, we note for further applications, that since $u(1)=0$ for $u \in \mathfrak{U}_{L}^{+}$it follows that for arbitrary $u \in \mathfrak{U}_{L}$ we have $u(1)=\epsilon(u)$. Indeed, $u(1)$ $=[u-\epsilon(u)](1)+[\epsilon(u)](1)=\epsilon(u)$.

The restricted cohomology groups $H_{*}^{n}(F, \&)$ were defined by Hochschild in [6], as the cohomology groups $H^{n}\left(\mathfrak{U}_{L}, F\right)$. In what follows we shall use the following definition of the cohomology groups $H^{n}\left(\mathfrak{U}_{L}, F\right)$ : Let $\operatorname{Hom}_{C}\left(\mathfrak{U}_{L}^{n}, F\right)$ be the additive group of all $C$-linear functions $f: \mathfrak{U}_{L} \otimes \cdots \otimes \mathfrak{U}_{L} \rightarrow F$. The sequence of these groups $\operatorname{Hom}_{C}\left(\mathfrak{U}_{L}^{n}, F\right)$ is a cochain complex with the coderivation $\left({ }^{10}\right)$ :

$$
\begin{aligned}
& \left(\delta^{n} f\right)\left(u_{1}, \cdots, u_{n+1}\right)=(-1)^{n+1} u_{1} f\left(u_{2}, \cdots, u_{n+1}\right) \\
& \quad+\sum_{i=1}^{n}(-1)^{n+1-i} f\left(u_{1}, \cdots, u_{i} u_{i+1}, \cdots\right)+f\left(u_{1}, \cdots, u_{n}\right) \epsilon\left(u_{n+1}\right),
\end{aligned}
$$

and $H_{*}^{n}(F, L)=H^{n}\left(\mathfrak{U}_{L} F\right)=$ Kernel $\delta^{n} /$ Image $\delta^{n-1}$.

Next we turn the ring $F^{n+1}$ into a $U_{L}^{n}$-module as follows:

For $v=U_{1} \otimes \cdots \otimes u_{n} \in \mathfrak{U}_{L}^{n}$ and $a=h_{0} \otimes \cdots \otimes h_{n}$, we set:

$$
\left(u_{1} \otimes \cdots \otimes u_{n}\right)(a)=h_{0}\left[u_{1}\left[h_{2} u_{2}\left[\cdots\left[h_{n-1} u_{n}\left(h_{n}\right)\right] \cdots\right]\right]\right] .
$$

For example,

$\left({ }^{10}\right)$ Note the difference in the sign $(-1)^{n+1}$ between this definition of $\delta^{n}$ and the classical definition of e.g. [6]. 


$$
u_{1}\left(h_{0} \otimes h_{1}\right)=h_{0} u_{1}\left(h_{1}\right), \quad\left(u_{1} \otimes u_{2}\right)\left(h_{0} \otimes h_{1} \otimes h_{2}\right)=h_{0}\left[u_{1}\left[h_{1} u_{2}\left(h_{2}\right)\right]\right], \text { etc. }
$$

and one extends the definition (7.5) linearly to all $a \in F^{n+1}$ and all $v \in \mathfrak{U}_{L}^{n}$. It is now easily verified that $F^{n+1}$ is a well defined $\mathfrak{U}_{L}^{n}$-module.

To any $a \in F^{n+1}$ we associate a function $f_{a} \in \operatorname{Hom}_{C}\left(\mathfrak{U}_{L}^{n}, F\right)$ defined as follows:

$$
f_{a}\left(u_{1}, \cdots, u_{n}\right)=\left(u_{1} \otimes \cdots \otimes u_{n}\right)(a), \quad u_{i} \in \mathfrak{U}_{L} .
$$

First we show:

Lemma 7.2. The mapping $\rho: a \rightarrow f_{a}$ is a monomorphism of $F^{n+1}$ into $\operatorname{Hom}_{C}\left(\mathfrak{U}_{L}^{n}, F\right)$ satisfying $\rho \Delta_{n+1}=\delta^{n} \rho$.

Since $f_{c a}=c f_{a}$ for $c \in C$ and $f_{a+b}=f_{a}+f_{b}$, it follows that $\rho$ is a homomorphism, and Corollary 7.1 (to be proved later) implies that $\rho$ is actually a monomorphism.

It remains now to prove that $\rho \Delta_{n+1}=\delta^{n} \rho$. It follows by definition of $\rho$ and of $\Delta_{n+1}$ that

$$
\left(\rho \Delta_{n+1}\right)(a)=\rho\left[\sum_{i=0}^{n+1}(-1)^{n+1-i} \epsilon_{i}(a)\right]=\sum_{i=0}^{n+1}(-1)^{n+1-i} f_{\epsilon_{i}(a)},
$$

and we first compute the functions $f_{\epsilon_{i}(a)}$ for elements $a \in F^{n+1}$ of the form $a=h_{0} \otimes h_{1} \otimes \cdots \otimes h_{n}=h_{0}^{(1)} h_{1}^{(2)} \cdots h_{n}^{(n+1)}$. We recall that $h^{(i)}=1 \otimes \cdots \otimes h$ $\otimes \cdots \otimes 1$, with $h$ standing in the $i$ th place.

It follows readily by the definition of $\epsilon_{i}=\epsilon_{F^{n+1-i}, i}$ that:

$$
\epsilon_{i}\left(h^{(j)}\right)=h^{(j)} \text { for } j<i \text {, and } \epsilon_{i}\left(h^{(j)}\right)=h^{(j+1)} \text { for } j>i .
$$

Hence, $\epsilon_{0}(a)=\epsilon_{0}\left(h_{0}^{(1)}\right) \epsilon_{0}\left(h_{1}^{(2)}\right) \cdots \epsilon_{0}\left(h_{n}^{(n+1)}\right)=h_{0}^{(2)} h_{1}^{(3)} \cdots h_{n}^{(n+2)}$, which implies, by (7.5), that:

$$
\begin{aligned}
f_{e_{0}(a)}\left(u_{1}, \cdots,\right. & \left.u_{n+1}\right) \\
& =u_{1}\left[h_{0} u_{2}\left[\cdots h_{n-1} u_{n+1}\left(h_{n+2}\right)\right] \cdots\right]=u_{1} f_{a}\left(u_{2}, \cdots, u_{n+1}\right) .
\end{aligned}
$$

If $i=1,2, \cdots, n$ then we obtain by (7.8) and (7.5) that:

$$
\epsilon_{i}(a)=\epsilon_{i}\left(h_{0}^{(1)}\right) \cdots \epsilon_{i}\left(h_{n}^{(n+1)}\right)=h_{0}^{(1)} \cdots h_{i-1}^{(i)} h_{i}^{(i+2)} \cdots h_{n}^{(n+2)} .
$$

Hence,

$$
\begin{aligned}
f_{\epsilon_{1}(a)}\left(u_{1}, \cdots, u_{n+1}\right) & \\
= & \left(u_{1} \otimes \cdots \otimes u_{n+1}\right)\left(h_{0}^{(1)} \cdots h_{i-1}^{(i)} h^{(i+2)} \cdots h_{n}^{(n+2)}\right) \\
= & h_{0} u_{1}\left[h_{1} \cdots u_{i-1}\left[h_{i-1} u_{i} u_{i+1}\left[h_{i} \cdots u_{n+1}\left(h_{n}\right)\right] \cdots\right]\right] \\
= & f_{a}\left(u_{1}, \cdots, u_{i} u_{i+1}, \cdots, u_{n+1}\right) .
\end{aligned}
$$

If $i=n+1$ then $\epsilon_{n+1}(a)=h_{0}^{(1)} h_{1}^{(2)} \cdots h_{n}^{(n)}$ and, therefore, 


$$
\begin{aligned}
f_{\epsilon_{n+1}(a)}\left(u_{1}, \cdots, u_{n+1}\right)=\left(u_{1} \otimes \cdots \otimes u_{n+1}\right)\left(h_{0}^{(1)} \cdots h_{n}^{(n+1)}\right) \\
\quad=h_{0}\left[u_{1}\left[h_{1} \cdots u_{n}\left[h_{n} u_{n+1}(1)\right] \cdots\right]\right]=f_{a}\left(u_{1}, \cdots, u_{n}\right) \epsilon\left(u_{n+1}\right),
\end{aligned}
$$

since it was shown that $u_{n+1}(1)=\epsilon\left(u_{n+1}\right) \in C$ and all the operators $u_{i}$ commute with the elements of the field $C$.

The mappings $\epsilon_{i}$ are isomorphisms and $f_{a+b}=f_{a}+f_{b}$ holds for all $a, b \in F^{n+1}$; hence, it follows that the three relations (7.9a), (7.9b) and (7.9c) hold for all $a \in F^{n+1}$.

The requirement that $\rho \Delta_{n+1}=\delta^{n} \rho$ is equivalent to the condition that:

$$
f_{\Delta_{n+1}(a)}=\left(\rho \Delta_{n+1}\right)(a)=\left(\delta^{n} \rho\right)(a)=\delta^{n} f_{a},
$$

and this is now readily obtained by inserting the three relations (7.9) into (7.7) and comparing the outcome with (7.4).

We intend now to show that $\rho$ induces an isomorphism of the cohomology groups $H^{n}\left(F^{+}\right)$into the restricted cohomology groups $H_{*}^{n}(F, \mathfrak{L})$. To this end we have to consider a certain base of $\mathscr{L}$ :

Let $(F: C)=p^{k}$, then it is known that $F=C\left[x_{1}, \cdots, x_{k}\right]$ where $x_{i}^{p}=c_{i} \in C$. Every $h \in F$ can be expressed uniquely in the form $h=f\left(x_{1}, \cdots, x_{k}\right)$ where $f$ is a polynomial in the $x$ 's of degree $<p$ in each $x_{i}$. We define a derivation $D_{i} \in \mathcal{L}$ of $F$ over $C$ by setting $D_{i}(h)=\partial f / \partial x_{i}$, where $\partial / \partial x_{i}$ is the formal partial derivation of polynomials $f$ with respect to the variable $x_{i}$. Evidently, $D_{i}$ is a well defined derivation and $D_{j}\left(x_{i}\right)=\delta_{i, j}$.

The derivations $D_{1}, \cdots, D_{k}$ are an $F$-base of $\mathcal{L}$. Indeed, if $D \in \mathcal{L}$ then $D_{0}=D-\sum D_{i}\left(x_{j}\right) D_{i}$ is also a derivation of $F$ and $D_{0}\left(x_{i}\right)=0$ for all $x_{i}$. This immediately implies that $D_{0}=0$, whence $D=\sum D_{i}\left(x_{j}\right) D_{i}$, and our assertion follows since the derivations $D_{i}$ are $F$-independent.

We shall denote by $\mathfrak{U}_{0}$ the $C$-subalgebra of $\mathfrak{U}_{L}$ generated by the derivations $D_{i}$. One readily verifies that $\mathfrak{U}_{0}$ is a commutative algebra and that $D_{i}^{p}=0$.

We introduce the following shortenings of notations: $(\nu)=\left(\nu_{1}, \cdots, \nu_{k}\right)$ be $k$-tuples of non-negative integers. We shall adopt the accepted definition of the sum of vectors: $\left(\nu_{i}\right)+\left(\mu_{i}\right)=\left(\nu_{i}+\mu_{i}\right)$.

If $a=\left(a_{1}, \cdots, a_{k}\right)$ is a vector of elements in any commutative ring then we set $a^{(v)}=a_{1}^{\nu_{1}} a_{2}^{\nu_{2}} \cdots a_{k}^{\nu_{k}}$ and $a^{(0)}=1$. One readily verifies the following rule :

$$
a^{(\nu)} \cdot a^{(\mu)}=a^{(\nu)+(\mu)} .
$$

In particular, for $D=\left(D_{1}, \cdots, D_{k}\right)$ we have $D^{(\nu)}=D_{1}^{\nu_{1}} \cdots D_{\boldsymbol{k}_{k}}^{\nu_{k}} \in \mathfrak{U}_{0}$ and $D^{(\nu)}=0$ if some $\nu_{i}>p$. It seems to be more convenient to consider the following elements of $\mathfrak{U}_{0}$ :

$$
D_{(v)}=\left(\nu_{1} ! \cdots \nu_{k} !\right)^{-1} D^{(\nu)} .
$$

Similarly to the Leibnitz' rule, one shows that:

$$
D_{(\nu)}(a b)=\sum_{(\lambda)+(\rho)=(\nu)} D_{(\lambda)}(a)_{(\rho)}(b) .
$$


For $h \in F$, we put $d_{j}(h)=h^{(j+1)}-h^{(j)}, j=1, \cdots, n$, and we recall again that $h^{(j)}=1 \otimes \cdots \otimes h \otimes \cdots \otimes 1$ with $h$ in the $j$ th place. Let $x=\left(x_{1}, \cdots, x_{k}\right)$ be the vector whose coordinates are the generators $x_{i}$ which were chosen above, then we set $d_{j}=d_{j}(x)=\left(d_{j}\left(x_{1}\right), \cdots, d_{j}\left(x_{k}\right)\right)$; and, thus, $d_{j}^{(\nu)}=d_{j}\left(x_{1}\right)^{\nu_{1}}$ $\cdots d_{j}\left(x_{k}\right)^{\nu k}$. These elements are of importance as seen in the following lemma:

Lemma 7.3. The elements $\left\{d_{1}^{\left(\nu_{1}\right)} d_{2}^{\left(\nu_{2}\right)} \cdots d_{n}^{\left(\nu_{n}\right)} ;\left(\nu_{i}\right)=\left(\nu_{i 1}, \cdots, \nu_{i k}\right), p>\nu_{i j}\right.$ $\geqq 0\}$ form an F-base of $F^{n-1}$, and for every $a \in F^{n+1}$ the following holds:

$$
a=\sum f_{a}\left(D_{\left(\nu_{1}\right)}, \cdots, D_{\left(\nu_{n}\right)}\right) d_{1}^{\left(\nu_{1}\right)} \cdots d_{n}^{\left(\nu_{n}\right)}
$$

where $f_{a}$ was defined in (7.6).

Proof. If $h \in F$, then $h=g\left(x_{1}, \cdots, x_{k}\right)$ is a polynomial of degree $<p$ in each $x_{i}$. Since $x_{i}^{(j+1)}=x_{i}^{(j)}+d_{j}\left(x_{i}\right)$, it follows by the Taylor expansion for polynomials that:

$$
\begin{aligned}
h^{(j+1)} & =f^{(j+1)}\left(x_{1}, \cdots, x_{k}\right)=f\left(x_{1}^{(j+1)}, \cdots, x_{k}^{(j+1)}\right) \\
& =f\left(x_{1}^{(j)}+d_{j}\left(x_{1}\right), \cdots, x_{k}^{(j)}+d_{j}\left(x_{k}\right)\right) \\
& \left.=\sum\left(\nu_{1} ! \cdots \nu_{k} !\right)^{-1}\left(\partial / \partial x_{1}^{\nu_{1}} \cdots \partial x_{k}^{\nu_{k}}\right) f\right]^{(j)} d_{j}^{\nu_{1}}\left(x_{1}\right) \cdots d_{j}^{\nu_{k}}\left(x_{k}\right) \\
& =\sum\left[D_{(\nu)}(h)\right]^{(j)} d_{j}^{(\nu)} .
\end{aligned}
$$

Namely,

$$
h^{(j+1)}=\sum\left[D_{(\nu)}(h)\right]^{(j)} d_{j}^{(\nu)} \text { for } h \in F .
$$

Hence,

$$
\left[D_{(\nu)}(h)\right]^{(j)}=\sum\left[D_{(\mu)}\left[D_{(\nu)}(h)\right]\right]^{(j-1)} d_{j-1}^{(\mu)}, \text { for } j>1,
$$

which implies that

$$
h^{(j-1)}=\sum_{(\nu),(\mu)}\left[D_{(\mu)}\left[D_{(\nu)}(h)\right]\right]^{(j-1)} d_{j-1}^{(\mu)} d_{j}^{(\nu)} .
$$

By repeating this process we obtain:

$$
\begin{aligned}
h^{(l)}=\sum\left[D_{\left(\nu_{1}\right)} D_{\left(\nu_{2}\right)} \cdots D_{\left(\nu_{l}\right)}(h)\right] d_{1}^{\left(\nu_{1}\right)} d_{2}^{\left(\nu_{2}\right)} \cdots d_{l}^{\left(\nu_{l}\right)} & \\
\text { for } l & =1,2, \cdots, n+1 .
\end{aligned}
$$

It follows now easily from (B) that every $a \in F^{n+1}$ can be expressed as a linear combination of the elements $\left\{d_{1}^{\left(\nu_{1}\right)} d_{2}^{\left(\nu_{2}\right)} \cdots d_{n}^{\left(\nu_{n}\right)}\right\}$ with coefficients in $F$. 
The number of the latter is exactly $\left(F^{n+1}: F\right)=p^{k n}$, since one has only to consider the $k$-tuples $\left(\nu_{i}\right)$ with $0 \leqq \nu_{i j}<p$; hence, these elements are an $F$-base of $F^{n+1}$, which proves the first part of the lemma.

Formula (B) which we have obtained above is exactly (7.11) for $a=h^{(l)}$. This is a consequence of (7.6), (7.5) and that:

$$
\left(D_{\left(\nu_{1}\right)} \otimes \cdots \otimes D_{\left(\nu_{n}\right)}\right)\left(h^{l}\right)=\left(D_{\left(\nu_{1}\right)} \cdots D_{\left(\nu_{l}\right)}\right)(h) \text { if }\left(\nu_{l+1}\right)=\cdots=\left(\nu_{n}\right)=(0)
$$

and zero otherwise.

Let $a=h_{0}^{(1)} \cdots h_{n}^{(n+1)} \in F^{n+1}$, then it follows by (B) that:

$$
\begin{aligned}
a & =h_{0}^{(1)}\left(\sum D_{\left(\nu_{1}\right)}\left(h_{1}\right) d_{1}^{\left(\nu_{1}\right)}\right) \cdots\left(\sum\left[D_{\left(\nu_{1}\right)} \cdots D_{\left(\nu_{n}\right)}\left(h_{n}\right)\right] d_{1}^{(\nu)} \cdots d_{n}^{\left(\nu_{n}\right)}\right) \\
& =\sum g_{a}\left(\left(\rho_{1}\right), \cdots,\left(\rho_{n}\right)\right) d_{1}^{\left(\rho_{1}\right)} \cdots d_{n}^{\left(\rho_{n}\right)}
\end{aligned}
$$

and

$$
g_{a}\left(\left(\rho_{1}\right), \cdots,\left(\rho_{n}\right)\right)=\sum h_{0} D_{\left(\nu_{1}^{1}\right)}\left(h_{1}\right)\left[D_{\left(\nu_{1}^{2}\right)} D_{\left(\nu_{2}{ }^{2}\right)}\left(h_{2}\right)\right] \cdots\left[D_{\left(\nu_{1}{ }^{n}\right)} \cdots D_{\left(\nu_{n}{ }^{n}\right)}\left(h_{n}\right)\right]
$$

where the sum ranges over all $k$-tuples $\left(\nu_{j}^{i}\right)$ such that:

$$
\left(\nu_{1}^{1}\right)+\cdots+\left(\nu_{1}^{n}\right)=\left(\rho_{1}\right), \cdots,\left(\nu_{i}^{i}\right)+\cdots+\left(\nu_{i}^{n}\right)=\left(\rho_{i}\right), \cdots,\left(\nu_{n}^{n}\right)=\left(\rho_{n}\right) .
$$

One verifies easily by ( $7.10 \mathrm{~b})$ that:

$$
\begin{aligned}
g_{a}\left(\left(\rho_{1}\right), \cdots,\left(\rho_{n}\right)\right)=h_{0} D_{\left(\rho_{1}\right)}\left[h_{1} D_{\left(\rho_{2}\right)}\left[h_{2} D_{\left(\rho_{3}\right)}\left[\cdots\left[h_{n-1} D_{\left(\rho_{n}\right)}\left(h_{n}\right)\right] \cdots\right]\right]\right] \\
\quad=\left(D_{\left(\rho_{1}\right)} \otimes \cdots \otimes D_{\left(\rho_{n}\right)}\right)\left(h_{0}^{(1)} h_{1}^{(2)} \cdots h_{n}^{(n+1)}\right)=f_{a}\left(D_{\left(\rho_{1}\right)}, \cdots, D_{\left(\rho_{n}\right)}\right),
\end{aligned}
$$

which proves the validity of (7.11) for elements $a=h_{0}^{(1)} h_{1}^{(2)} \cdots h_{n}^{(n+1)}$. The rest follows now from the fact that if (7.11) holds for two elements $a, b$ then it is valid also for the element $a+b$.

A simple consequence of (7.11) is the following:

Corollary 7.1. $a=0$ if and only if $f_{a}\left(D_{\left(\nu_{1}\right)}, \cdots, D_{\left(\nu_{n}\right)}\right)=0$ for all $D_{\left(\nu_{n}\right)} \in U_{0}$.

We are now in position to deal with an inverse homomorphism of the homomorphism $\rho$ given in Lemma 7.2:

For any function $f \in \operatorname{Hom}_{C}\left(\mathfrak{U}_{L}^{n}, F\right)$ we shall denote by $\sigma(f)=a_{f}$ an element of $F^{n+1}$ defined as follows:

$$
a_{f}=\sum f\left(D_{\left(\nu_{1}\right)}, \cdots, D_{\left(\nu_{n}\right)}\right) d_{1}^{\left(\nu_{1}\right)} \cdots d_{n}^{\left(\nu_{n}\right)} .
$$

Then:

Lemma 7.4. The mapping $\sigma: f \rightarrow a_{f}$ is an epimorpism of $\operatorname{Hom}_{C}\left(U_{L}^{n}, F\right)$ on $F^{n+1}$ satisfying: (1) $\sigma \rho=i d e n t i t y,(2)$ The restrictions of $\rho \sigma(f)$ and $f$ on $U_{0}^{n}$ coincide: (3) $\sigma \delta_{1}^{n_{1}}=\Delta_{n+1} \sigma$. 
Proof. It follows from (7.12) that $\sigma$ is a homomorphism, and (7.11) implies that it is an epimorpism. Furthermore, the uniqueness of (7.11) implies by comparing it with (7.12) that $\sigma \rho(a)=a$ for $a \in F^{n+1}$, which proves (1). Indeed,

$$
\begin{aligned}
\sigma \rho(a) & =\sum \rho(a)\left(D_{\left(\nu_{1}\right)}, \cdots, D_{\left(\nu_{n}\right)}\right) d_{1}^{\left(\nu_{1}\right)} \cdots d_{n}^{\left(\nu_{n}\right)} \\
& =\sum f_{a}\left(D_{\left(\nu_{1}\right)}, \cdots, D_{\left(\nu_{n}\right)}\right) d_{1}^{\left(\nu_{1}\right)} \cdots d_{n}^{\left(\nu_{n}\right)}=a .
\end{aligned}
$$

The same reason yields that $f \sigma_{(a)}\left(D_{\left(\nu_{1}\right)}, \cdots, D_{\left(\nu_{n}\right)}\right)=f\left(D_{\left(\nu_{1}\right)}, \cdots, D_{\left(v_{n}\right)}\right)$ for all $D_{(v)} \in \mathfrak{H}_{0}$, which readily implies that the restrictions of $\rho \sigma(a)=f_{\sigma(a)}$ and $f$ on $\mathfrak{U}_{0}^{n}$ coincide.

To prove (3) of Lemma 7.4, we observe that $\sigma(f)=\sigma(g)$ if and only if $f_{0}=g_{0}$, where $f_{0}$ denotes the restriction of $f \in \operatorname{Hom}_{C}\left(\mathfrak{U}_{L}^{n}, F\right)$ to $\mathfrak{u}_{0}^{n}$. With this notation, condition (2) of our lemma means that $[\rho \sigma(f)]_{0}=f_{0}$. One also verifies easily that if $f_{0}=g_{0}$ then $\left(\delta^{n} f\right)_{0}=\left(\delta^{n} g\right)_{0}$. Hence, it follows from (2) that $\left[\left(\delta^{n} \rho \sigma\right)(f)\right]_{0}=\left[\delta^{n} f\right]_{0}$, which implies by the preceding remarks that $\sigma \delta^{n} \rho \sigma=\sigma \delta^{n}$. It follows, therefore, by (1) of our lemma and by Lemma 7.2 that:

$$
\sigma \delta^{n}=\sigma \delta^{n} \rho \sigma=\sigma \rho \Delta_{n+1} \sigma=\Delta_{n+1} \sigma . \text { q.e.d. }
$$

Combining now the results of Lemma 7.2 and Lemma 7.4 we obtain the following situation: The homomorphism $\rho$ of Lemma 7.2 induces a homomorphism $\rho^{*}$ of $H^{n}\left(F^{+}\right)$into $H^{n}\left(\mathfrak{U}_{L}, F\right)$; on the other hand, the homomorphism $\sigma$ of Lemma 7.4 induces a homomorphism $\sigma^{*}$ of $H^{n}\left(\mathfrak{U}_{L}, F\right)$ and (1) of Lemma 7.4 implies that $\sigma^{*} \rho^{*}=$ identity mapping of $H^{n}\left(F^{+}\right)$. From which one readily verifies:

THEOREM 7.3. $\rho^{*}$ is an isomorphism of $H^{n}\left(F^{+}\right)$into $H_{*}^{n}(F, \mathfrak{L})=H^{n}\left(\mathfrak{U}_{L}, F\right)$, and $\rho^{*}\left[\left(H^{n}\left(F^{+}\right)\right]\right.$is a direct summand of $H_{*}^{n}(F, \mathfrak{L})$, i.e. $H_{*}(F, \mathfrak{L})=\rho^{*}\left[H^{n}\left(F^{+}\right)\right]$ $\oplus \sigma^{*-1}(0)$.

In particular, it follows by Theorem 7.2 and Theorem 5.4 that:

THEOREm 7.4. The Brauer group $\mathfrak{B}(F)$ of all central simple algebras over $C$ which are split by $F$ is isomorphic with a subgroup of the restricted cohomology group $H_{*}^{2}(\mathcal{L}, F)$.

A result of this type was obtained by Hochschild in [7] and probably the canonical map of $\operatorname{Ext}(F, \mathfrak{L})$ into $H^{2}(F, \mathfrak{L})$ maps the "regular extensions" of $\mathcal{L}$ by $F$, which were introduced by Hochschild in [7], onto the group $\rho^{*}\left[H^{2}\left(F^{+}\right)\right]$, but we were unable to carry out the complicated computations involved in proving this result.

In the proof of Lemma 7.4 we have observed that $\sigma(f)=\sigma(g)$ if and only if the restriction $f_{0}, g_{0}$ of $f$ and $g$, respectively, on $U_{0}^{n}$ coincide. It follows, therefore, that $\sigma$ induces an isomorphism of $\operatorname{Hom}_{C}\left(\mathfrak{U}_{0}^{n}, F\right)$ onto $F^{n+1}$, and, thus, Lemma 7.4 implies that $\sigma$ induces also the following isomorphism: 
Theorem 7.5. $H^{n}\left(F^{+}\right) \cong H^{n}\left(\mathfrak{U}_{0}, F\right)$.

One still would like to have an invariant characterization of the image $\rho^{*}\left(H^{n}\left(F^{+}\right)\right)$.

8. An application. In the present section we apply the preceding theory of the representation of the Brauer group to prove another result of Hochschild [8] with a slight generality.

Let $F$ be the algebraic closed extension of a field $C$ of characteristic $p \neq 0$. Let $\mathfrak{B}(F / C)$ denote the Brauer group of all finite dimensional central simple algebras over $C$ (and, evidently, split by $F$ ). Let $\mathfrak{e}(F / C)$ be the complex:

$$
1 \rightarrow F^{*} \rightarrow\left(F \otimes_{C} F\right)^{*} \rightarrow \cdots \rightarrow\left(F \otimes_{C} F \otimes_{C} \cdots \otimes_{C} F\right)^{*} \rightarrow .
$$

The cohomology groups of this complex will be denoted by $H^{n}(F / C)$, since we want to emphasize the role of the field $C$.

In view of Remark 5.2 and Theorem 5.4, it follows that $H^{2}(F / C) \cong \mathfrak{B}(F / C)$.

Consider now the mapping $\pi^{-1}: a \rightarrow a^{1 / p}$ for $a \in F \otimes_{C} \cdots \otimes_{C} F$. Since $\pi^{-1}$ is an automorphism of $F$ and $\pi^{-1}$ maps $C$ onto $C^{1 / p}$ it follows that $\pi^{-1}$ maps $F \otimes_{C} \cdots \otimes_{C} F$ onto $F \otimes_{C^{1 / p}} \cdots \otimes_{C^{1 / p}} F$. Hence, $\pi^{-1}$ maps isomorphically the groups of the complex $\mathfrak{e}(F / C)$ onto $\mathfrak{e}\left(F / C^{1 / p}\right)$. Furthermore, it follows immediately from the definition of $\epsilon_{i}$ in (5.2) and the definition of $\Delta_{n}$ in (5.3), that $\pi \Delta=\Delta \pi$. Consequently:

THEOREM 8.1. The automorphism $\pi: a \rightarrow a^{1 / p}$ induces an isomorphism $H^{n}(F / C) \cong H^{n}\left(F / C^{1 / p}\right)$.

In particular, it follows for $n=2$ that:

Theorem 8.2. $\mathfrak{B}(F / C) \cong \mathfrak{B}\left(F / C^{1 / p}\right)$.

A more detailed study of this mapping gives more information on the isomorphism between the Brauer group $\mathfrak{B}(F / C)$ of all central simple algebras over $C$ and the Brauer group $\mathfrak{B}\left(F / C^{1 / p}\right)$ of all algebras over $C^{1 / p}$, and first we prove:

Lemma 8.1. Every algebra $\mathfrak{A}$ over $C$ is similar (in the sense of Brauer) to an algerbra $\mathfrak{B}^{p}$.

Proof. Let $a \in H^{2}(F / C)$ which corresponds to $A$. From the definition of the complex $\mathrm{C}(F)$ and the mappings $\epsilon_{F^{n-i}, i}$ and $\Delta_{n}$ in (5.2), (5.3), it is easily verified that $a^{1 / p} \in H^{2}(F / C)$. Let $\mathfrak{B}$ be the central simple algebra corresponding to $a^{1 / p}$, then one concludes from the isomorphism $H^{2}(F / C) \cong \mathfrak{B}(F / C)$ that $\mathfrak{B}^{p}$ is similar to $\mathfrak{A}$.

To determine the isomorphism of Theorem 8.2, we observe that if $a \in H^{2}(F / C)$ and corresponds to $\mathfrak{A}$, then one has to consider $a^{1 / p}$ in that theorem not as $\in H^{2}(F / C)$ but as a cocycle of $H^{2}\left(F / C^{1 / p}\right)$ to which corresponds an algebra $\mathfrak{A}_{1 / p}$ over $C^{1 / p}$. It is not difficult to show that the inclusion 
mapping of the cocycles of $H^{2}(F / C)$ into $H^{2}\left(F / C^{1 / p}\right)$ is equivalent to the correspondence of the algebras $\mathfrak{A}$ over $C$ to their field extension $\mathfrak{A} \otimes C^{1 / p}$ over $C^{1 / p}$. Hence, in our case we have that $\mathfrak{A}_{1 / p}$ is similar to the algebra $\mathfrak{B} \otimes C^{1 / p}$, where $\mathfrak{B}^{p} \cong \mathfrak{A}$ and both correspond to the cocycle $a^{1 / p}$.

Thus, we have shown that the isomorphism of Theorem 8.2 is the correspondence $\mathfrak{A} \rightarrow \mathfrak{B} \otimes_{C} C^{1 / p}$ where $\mathfrak{B}^{p} \cong \mathfrak{A}$. Since this mapping maps $\mathfrak{B}(F / C)$ onto $\mathfrak{B}\left(F / C^{1 / p}\right)$ and $\mathfrak{B}$ is an algebra over $C$, it follows:

THeOREM 8.3. Every central simple algebra $D$ over $C^{1 / p}$ is similar to an algebra $\mathfrak{D}_{0} \otimes C^{1 / p}$ where $\mathfrak{D}_{0}$ is a central simple algebra over $C$.

This result, in a different version, has been obtained by Hochschild in [8] using a different method.

\section{BIBLIOGRAPHY}

1. S. A. Amitsur, Generic splitting fields of central simple algebras, Ann. of Math. vol. 62 (1955) pp. 8-43.

2. - Some results on central simple algebras, Ann. of Math. vol. 63 (1956) pp. 285-293.

3. E. Artin, Zur theorie der hyperkomplexen Zahlen, Abh. Math. Sem. Univ. Hamburg. vol. 5 (1927) pp. 245-250.

4. H. Cartan and E. Eilenberg, Homological algebra, Princeton University Press, Princeton, 1956.

5. G. Hochschild, Double vector spaces over division rings, Amer. J. Math. vol. 71 (1949) pp. 443-460.

6. - Cohomology of restricted Lie algebras, Amer. J. Math. vol. 76 (1954) pp. 555-580.

7. - Simple algebras with purely inseparable splitting fields of exponent 1, Trans. Amer. Math. Soc. vol. 79 (1955) pp. 477-489.

8. - Restricted Lie algebras and simple associative algebras of characteristic $p$, Trans. Amer. Math. Soc. vol. 80 (1955) pp. 135-147.

9. N. Jacobson, Abstract derivations and Lie algebras, Trans. Amer. Math. Soc. vol. 42 (1937) pp. 206-224.

10. - An extension of Galois theory to non-normal and non-separable fields, Amer. J. Math. vol. 69 (1947) pp. 27-36; vol. 80 (1955) pp. 135-147.

11. T. Nakayama, Non-normal Galois theory for non-commutative and non-semi-simple rings, Canad. J. Math. vol. 3 (1951) pp. 208-216.

12. — Double vector spaces over arbitrary rings, Amer. J. Math. vol. 74 (1952) pp. 645655.

HEBREW UNIVERSITY, JERUSALEM, IsRaEL. 